Draft version December 12, 2019

Typeset using LATEX twocolumn style in AASTeX61

\title{
DETAILED ABUNDANCES IN THE ULTRA-FAINT MAGELLANIC SATELLITES CARINA II AND III*
}

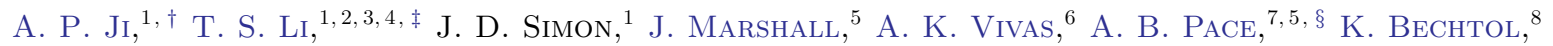 \\ A. Drlica-Wagner, ${ }^{3,4,9}$ S. E. Koposov,${ }^{7,10}$ T. T. Hansen, ${ }^{5}$ S. Allam ${ }^{11}$ R. A. Gruendl, ${ }^{12,13}$ M. D. Johnson, ${ }^{13}$ \\ M. McNanna, ${ }^{8}$ N. E. D. NoËL,${ }^{14}$ D. L. Tucker, ${ }^{11}$ And A. R. Walker ${ }^{6}$
}

(MagLites Collaboration)

\footnotetext{
${ }^{1}$ Observatories of the Carnegie Institution for Science, 813 Santa Barbara St., Pasadena, CA 91101, USA

${ }^{2}$ Department of Astrophysical Sciences, Princeton University, Princeton, NJ 08544, USA

${ }^{3}$ Fermi National Accelerator Laboratory, P.O. Box 500, Batavia, IL 60510, USA

${ }^{4}$ Kavli Institute for Cosmological Physics, University of Chicago, Chicago, IL 60637, USA

${ }^{5}$ George P. and Cynthia Woods Mitchell Institute for Fundamental Physics and Astronomy, and Department of Physics and Astronomy, Texas A\&M University, College Station, TX 77843, USA

${ }^{6}$ Cerro Tololo Inter-American Observatory, NSF's National Optical-Infrared Astronomy Research Laboratory, Casilla 603, La Serena, Chile

${ }^{7}$ McWilliams Center for Cosmology, Carnegie Mellon University, 5000 Forbes Ave, Pittsburgh, PA 15213, USA

${ }^{8}$ Physics Department, University of Wisconsin-Madison, 1150 University Avenue Madison, WI 53706, USA

${ }^{9}$ Department of Astronomy and Astrophysics, University of Chicago, Chicago IL 60637, USA

${ }^{10}$ Institute of Astronomy, University of Cambridge, Madingley Road, Cambridge CB3 OHA, UK

${ }^{11}$ Fermi National Accelerator Laboratory, P.O. Box 500, Batavia, IL 60510, USA

${ }^{12}$ Department of Astronomy, University of Illinois, 1002 W. Green Street, Urbana, IL 61801, USA

${ }^{13}$ National Center for Supercomputing Applications, 1205 West Clark St., Urbana, IL 61801, USA

${ }^{14}$ Department of Physics, University of Surrey, Guildford, GU2 7XH, UK
}

\section{Submitted to ApJ}

\begin{abstract}
We present the first detailed elemental abundances in the ultra-faint Magellanic satellite galaxies Carina II (Car II) and Carina III (Car III). With high-resolution Magellan/MIKE spectroscopy, we determined abundances of nine stars in Car II including the first abundances of an RR Lyrae star in an ultra-faint dwarf galaxy; and two stars in Car III. The chemical abundances demonstrate that both systems are clearly galaxies and not globular clusters. The stars in these galaxies mostly display abundance trends matching those of other similarly faint dwarf galaxies: enhanced but declining $[\alpha / \mathrm{Fe}]$ ratios, iron-peak elements matching the stellar halo, and unusually low neutron-capture element abundances. One star displays a low outlying $[\mathrm{Sc} / \mathrm{Fe}]=-1.0$. We detect a large Ba scatter in Car II, likely due to inhomogeneous enrichment by low-mass AGB star winds. The most striking abundance trend is for $[\mathrm{Mg} / \mathrm{Ca}]$ in Car II, which decreases from +0.4 to -0.4 and indicates clear variation in the initial progenitor masses of enriching core-collapse supernovae. So far, the only ultra-faint dwarf galaxies displaying a similar $[\mathrm{Mg} / \mathrm{Ca}]$ trend are likely satellites of the Large Magellanic Cloud. We find two stars with $[\mathrm{Fe} / \mathrm{H}] \leq-3.5$, whose abundances likely trace the first generation of metal-free Population III stars and are well-fit by Population III core-collapse supernova yields. An appendix describes our new abundance uncertainty analysis that propagates line-by-line stellar parameter uncertainties.
\end{abstract}

Keywords: stars: abundances — galaxies: dwarf — Local Group

Corresponding author: A. P. Ji

aji@carnegiescience.edu

\footnotetext{
* This paper includes data gathered with the 6.5 meter Magellan Telescopes located at Las Campanas Observatory, Chile.

$\dagger$ Hubble Fellow

$\ddagger$ NHFP Einstein Fellow

$\S$ Mitchell Astronomy Fellow
} 


\section{INTRODUCTION}

Ultra-faint dwarf galaxies (UFDs) are the luminous counterparts to the least massive star-forming dark matter halos, likely forming stars during the first $~ 1$ Gyr before being quenched by reionization (e.g., Bullock et al. 2000; Benson et al. 2002; Simon \& Geha 2007; Brown et al. 2014; Simon 2019). As a result, the chemical abundances of stars in UFDs preserve a clean snapshot of chemical enrichment from the earliest stages of galaxy formation and reionization, providing a window to the most metal-poor stellar populations and their nucleosynthetic output (Kirby et al. 2008; Frebel \& Bromm 2012; Geha et al. 2013; Weisz et al. 2014; Wise et al. 2014; Ji et al. 2015). Dozens of UFDs have now been discovered in deep, wide, and uniform photometric surveys such as the Sloan Digital Sky Survey, Pan-STARRS, and the Dark Energy Survey (DES) (e.g., Willman et al. 2005; Belokurov et al. 2007; Laevens et al. 2015; Bechtol et al. 2015; Koposov et al. 2015a; Drlica-Wagner et al. 2015). The large number of UFDs provides a large population of local objects that retain signatures of high-redshift star and galaxy formation.

Until recently, these UFDs have generally been assumed to be satellites of the Milky Way. However, the two most massive dwarfs orbiting the Milky Way, the Large and Small Magellanic Clouds (LMC and SMC), should have had their own satellite UFDs (e.g., D'Onghia \& Lake 2008; Koposov et al. 2015b; DrlicaWagner et al. 2015; Jethwa et al. 2016; Dooley et al. 2017; Sales et al. 2017). Since the LMC and SMC are likely on their first infall into the Milky Way (Besla et al. 2007; Busha et al. 2011; Kallivayalil et al. 2013; Simon 2018; Fritz et al. 2019; Pace \& Li 2019), any dwarfs that were previously Magellanic satellites could now be in the process of accretion into the Milky Way. Gaia proper motion measurements have revealed that several UFDs are kinematically associated with the LMC/SMC system (Kallivayalil et al. 2018; Erkal \& Belokurov 2019). Two of these LMC satellites are Carina II (Car II, $M_{V}=-4.5, L / L_{\odot} \sim 10^{3.7}$ ) and Carina III (Car III, $M_{V}=-2.4, L / L_{\odot} \sim 10^{2.9}$ ), discovered in the Magellanic Satellites Survey (MagLiteS, Drlica-Wagner et al. 2016; Torrealba et al. 2018) with the Dark Energy Camera (DECam, Flaugher et al. 2015) on the Blanco telescope. Li et al. (2018) spectroscopically confirmed Car II to be a dwarf galaxy, and Li et al. (in prep) have now confirmed Car III as a dwarf galaxy as well. These UFDs are only $\sim 20 \mathrm{kpc}$ away from the LMC, and are also close to the Sun (37.4 and $27.8 \mathrm{kpc}$ for Car II and III, respectively). Thus, they have a relatively large number of bright stars amenable for high-resolution spectroscopic followup and chemical abundance measurements.
In this paper, we present a comprehensive chemical abundance analysis of Magellan/MIKE spectroscopy of 9 stars in Car II and 2 stars in Car III. Along with Horologium I (Nagasawa et al. 2018), these are currently the only ultra-faint LMC satellites with high-resolution abundance measurements. Section 2 explains the observations, data reduction, and velocity measurements. Section 3 details our abundance analysis. We discuss the formation history of these galaxies in Section 4, highlighting the interesting $\alpha$-element abundance trends in Section 4.3. We focus on potential signatures of metalfree Pop III stars in Section 5, then summarize and conclude in Section 6.

\section{OBSERVATIONS, DATA REDUCTION, RADIAL VELOCITIES}

Our Carina II and III targets were selected to be the brightest radial velocity members from Magellan/IMACS, AAT/AAO, and VLT/FLAMES moderate resolution spectra, including five bright member stars from Li et al. (2018) and five new bright member stars from $\mathrm{Li}$ et al. (in prep). In addition, we include one RR Lyrae member in Carina II identified in Torrealba et al. (2018). We observed these stars with Magellan/MIKE (Bernstein et al. 2003) over four separate runs (Tables 1 and 2). Slits of width $0.5,0.7$, and $1 . .0$ were used depending on the seeing, resulting in typical resolutions of $R \sim 50 \mathrm{k} / 40 \mathrm{k}, 35 \mathrm{k} / 28 \mathrm{k}$, and $28 \mathrm{k} / 22 \mathrm{k}$ on the blue/red arms of MIKE, respectively. We used 2x2 binning for the $0 . .7$ and 1 .'0 slits, and $2 \times 1$ binning for the 0.5 slit. The MIKE data were reduced with CarPy (Kelson 2003).

We used the code SMHR (Casey 2014) ${ }^{1}$ to coadd, normalize, stitch orders, and Doppler correct the reduced spectra for abundance analysis. Data from multiple runs were combined by coadding order-by-order, using a common set of spline knot locations and line masks after adjusting for observed radial velocity. The signal-to-noise at the order center closest to rest wavelengths of $4500 \AA$, $5300 \AA$, and $6500 \AA$ is given in Table 1 . The total integrated time spent on these stars is 34 hours. Note there is significant reddening towards Car II and III $(\mathrm{E}(B-V)$ $\sim 0.2 \mathrm{mag})$. Figure 1 shows our spectra around the $\mathrm{C}-\mathrm{H}$ $\mathrm{G}$ band, the strongest barium line, and the $\mathrm{Mg} \mathrm{b}$ triplet.

In general, we reduced all MIKE data from a given observing run together before measuring the radial velocity. The exception is the RR Lyrae (RRL) star CarII-V3, which experiences large radial velocity variations on a short timescale. Using the known pulsation phases (Tor-

\footnotetext{
1 https://github.com/andycasey/smhr, first described in Casey 2014
} 

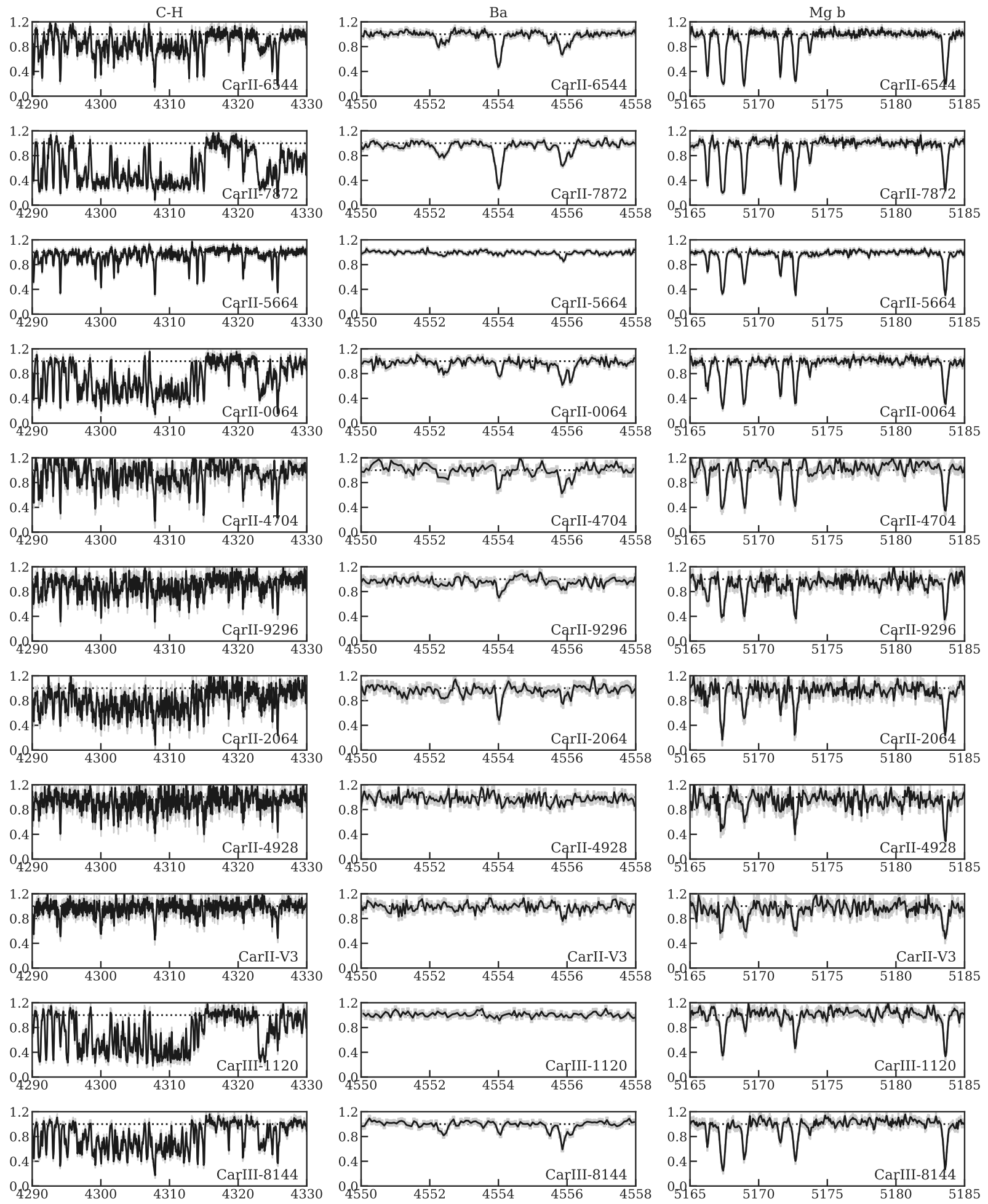

Figure 1. Spectrum of all stars around the $\mathrm{C}-\mathrm{H} \mathrm{G}$ band, the $\mathrm{Ba} 4554$ line, and the $\mathrm{Mg} \mathrm{b}$ lines. Stars are sorted in order of system and then increasing $T_{\text {eff }}$ from top to bottom (same as Table 3 ). Grey band indicates $\pm 1 \sigma$ spectrum noise. 
Table 1. Observations

\begin{tabular}{|c|c|c|c|c|c|c|c|c|c|c|}
\hline Star & source_id & RA & Dec & $\begin{array}{c}G \\
(\mathrm{mag})\end{array}$ & $\begin{array}{c}g_{0} \\
(\mathrm{mag})\end{array}$ & $\begin{array}{c}r_{0} \\
(\mathrm{mag})\end{array}$ & Slit & $\begin{array}{c}t_{\exp } \\
\text { (hour) }\end{array}$ & $\begin{array}{c}\text { SNR } \\
(4500 \AA)\end{array}$ & $\begin{array}{c}\text { SNR } \\
(6500 \AA ̊)\end{array}$ \\
\hline CarII-6544 & 5293947247051916544 & $07: 36: 51.11$ & $-58: 01: 46.3$ & 15.07 & 15.63 & 14.63 & 0.5 & 1.8 & 22 & 67 \\
\hline CarII-7872 & 5293894539213647872 & $07: 36: 51.89$ & $-58: 16: 39.2$ & 15.50 & 15.92 & 15.01 & 0.7 & 1.0 & 25 & 60 \\
\hline CarII-5664 & 5293896360279425664 & $07: 38: 08.51$ & $-58: 09: 35.0$ & 16.33 & 16.55 & 15.86 & 0.7 & 3.8 & 38 & 80 \\
\hline CarII-0064 & 5293951473299720064 & $07: 36: 21.25$ & $-57: 58: 00.2$ & 16.78 & 16.96 & 16.30 & $1^{\prime \prime} 0$ & 2.6 & 22 & 54 \\
\hline CarII-4704 & 5293928074318184704 & $07: 35: 37.66$ & $-58: 01: 51.8$ & 17.40 & 17.46 & 16.93 & 0.7 & 3.3 & 13 & 34 \\
\hline CarII-9296 & 5293900827045399296 & $07: 37: 39.79$ & $-58: 05: 06.9$ & 17.72 & 17.86 & 17.29 & $1^{\prime \prime} 0$ & 3.0 & 15 & 35 \\
\hline CarII-2064 & 5293951881319592064 & $07: 36: 01.33$ & $-57: 58: 43.8$ & 18.22 & 18.27 & 17.77 & $0 . \prime 7$ & 4.6 & 13 & 31 \\
\hline CarII-4928 & 5293951503362524928 & $07: 36: 24.98$ & $-57: 57: 14.2$ & 18.42 & 18.40 & 17.96 & $1^{\prime \prime} 0$ & 5.5 & 13 & 31 \\
\hline CarII-V3* & 5293940924860019584 & 07:35:09.12 & $-57: 57: 14.8$ & 18.46 & 18.13 & 18.01 & $1^{\prime \prime} 0$ & 2.5 & 15 & 24 \\
\hline CarIII-1120 & 5293955665187701120 & $07: 38: 22.30$ & $-57: 53: 02.1$ & 17.46 & 17.51 & 16.97 & 0.77 & 2.7 & 18 & 39 \\
\hline CarIII-8144 & 5293907630273478144 & $07: 38: 34.93$ & $-57: 57: 05.3$ & 17.65 & 17.72 & 17.18 & $0 ! 7$ & 3.2 & 21 & 41 \\
\hline
\end{tabular}

Note- Our star ID numbers are the last four digits of the Gaia source_id. $G$ is Gaia magnitudes. $g_{0}$ and $r_{0}$ are dereddened DECam photometry from MagLiteS, taken from Li et al. (in prep). SNR is per pixel

* This star is a variable RR Lyrae star. The magnitudes here are the mean magnitudes found by MagLiteS and Gaia (Torrealba et al. 2018; Clementini et al. 2019), where the DECam magnitudes have been dereddened. 
Table 2. Radial Velocities

\begin{tabular}{lccccc}
\hline \hline Star & Obs Date & MJD & $v_{\text {hel }}$ & $N_{\text {ord }}$ & $\sigma_{\text {sys }}$ \\
\hline CarII-6544 & $2018-01-24$ & 58142.031 & 470.4 & 34 & 1.2 \\
CarII-7872 & $2018-11-13$ & 58435.277 & 478.5 & 35 & 0.5 \\
CarII-5664 & $2018-11-16$ & 58438.207 & 483.7 & 34 & 0.6 \\
CarII-0064 & $2017-12-06$ & 58093.336 & 475.0 & 35 & 0.7 \\
CarII-4704 & $2018-11-13$ & 58435.319 & 472.4 & 30 & 1.0 \\
CarII-9296 & $2018-01-24$ & 58142.059 & 481.9 & 30 & 1.2 \\
CarII-2064 & $2018-01-24$ & 58142.210 & 473.9 & 34 & 0.9 \\
CarII-4928 & $2018-01-23$ & 58141.220 & 476.1 & 23 & 1.4 \\
CarII-V3 & $2018-11-15$ & 58437.247 & 478.2 & 18 & 1.9 \\
CarIII-1120 & $2018-01-24$ & 58142.147 & 283.7 & 30 & 1.1 \\
CarIII-8144 & $2018-11-16$ & 58438.277 & 280.8 & 36 & 0.5 \\
\hline
\end{tabular}

Note-We show one representative velocity measurement per star in our sample. The full table is available online. Note that CarII-6544 is likely a binary star and CarII-V3 is an RRL star, so these have significant velocity variations.

realba et al. 2018), we observed CarII-V3 across phases $0.40-0.55$ with five consecutive 30 -min exposures. Over this phase range, the star has fairly consistent stellar parameters (For et al. 2011), so individual exposures can be coadded after correcting for a velocity offset. We reduced each exposure separately, measured radial velocities for each observation separately using the $\mathrm{Mg} \mathrm{b}$ triplet, corrected each order to rest frame, and coadded order-by-order before stitching orders in SMHR.

Radial velocities are given in Table 2. For the velocity measurements, we re-reduced each exposure individually with CarPy. We measured radial velocities of the 40 orders from $3900 \AA$ to $6800 \AA$ (order numbers $51-90$ ). Of these, we masked the telluric lines around $6300 \AA$, discarded three orders from $5820-6020 \AA$ because of interstellar $\mathrm{Na} \mathrm{D}$ absorption, and discarded the bluest order on the red side due to uniformly low $\mathrm{S} / \mathrm{N}$. We crosscorrelated individual orders of our MIKE spectra against a normalized high-S/N MIKE spectrum of HD122563. To remove outliers, we iteratively sigma clip orders with velocities that are more than 5 biweight scales away from the biweight average. The final number of orders for each spectrum is given by $N_{\text {ord }}$ in Table 2. Statistical errors for each order were then found by calculating the $\chi^{2}$ at different velocities and taking $\Delta \chi^{2}=1$ away from the minimum.

Naively, we could combine these measurements by taking a weighted average of all orders to get a final average velocity and in principle reaching an extremely high velocity precision of $\sim 0.1 \mathrm{~km} / \mathrm{s}$. However, systematic ef-
Table 3. Stellar Parameters

\begin{tabular}{lcccc}
\hline \hline Star & $T_{\text {eff }}(\mathrm{K})$ & $\log g(\mathrm{dex})$ & $\nu_{t}\left(\mathrm{~km} \mathrm{~s}^{-1}\right)$ & {$[\mathrm{M} / \mathrm{H}]$} \\
\hline CarII-6544 & $4330 \pm 152$ & $0.40 \pm 0.31$ & $2.75 \pm 0.26$ & $-2.65 \pm 0.09$ \\
CarII-7872 & $4380 \pm 155$ & $0.75 \pm 0.32$ & $2.32 \pm 0.27$ & $-2.48 \pm 0.11$ \\
CarII-5664 & $4430 \pm 155$ & $0.45 \pm 0.31$ & $2.34 \pm 0.25$ & $-3.50 \pm 0.06$ \\
CarII-0064 & $4630 \pm 153$ & $1.15 \pm 0.32$ & $2.31 \pm 0.27$ & $-2.20 \pm 0.07$ \\
CarII-4704 & $4720 \pm 160$ & $1.30 \pm 0.31$ & $1.97 \pm 0.27$ & $-2.19 \pm 0.09$ \\
CarII-9296 & $4810 \pm 205$ & $1.40 \pm 0.37$ & $1.90 \pm 0.34$ & $-2.87 \pm 0.15$ \\
CarII-2064 & $5300 \pm 200$ & $2.70 \pm 0.35$ & $2.15 \pm 0.32$ & $-2.35 \pm 0.17$ \\
CarII-4928 & $5065 \pm 236$ & $2.35 \pm 0.46$ & $2.10 \pm 0.34$ & $-3.00 \pm 0.20$ \\
CarII-V3 & $6100 \pm 330$ & $1.75 \pm 0.27$ & $3.20 \pm 0.28$ & $-2.70 \pm 0.21$ \\
CarIII-1120 & $4500 \pm 216$ & $1.50 \pm 0.34$ & $1.85 \pm 0.32$ & $-3.89 \pm 0.14$ \\
CarIII-8144 & $4990 \pm 162$ & $2.20 \pm 0.32$ & $1.75 \pm 0.27$ & $-2.25 \pm 0.08$ \\
\hline
\end{tabular}

fects dominate both the velocity measurement and error. For example, MIKE is not attached to the instrument rotator and until recently did not have an atmospheric dispersion compensator. At high airmasses, atmospheric refraction in the narrow slit direction causes systematic velocity offsets as a function of wavelength that can be as large as $2-3 \mathrm{~km} / \mathrm{s}$. We will correct for these effects in later work, but such velocity differences do not impact the abundance analyses that are the focus of this paper. Thus, for now in Table 2 we provide the radial velocity of each individual spectrum computed by an inverse-variance weighted average of all $N_{\text {ord }}$ orders. The systematic error is the weighted standard deviation of those orders and dominates over the $\sim 0.1 \mathrm{~km} / \mathrm{s}$ statistical uncertainty.

\section{ABUNDANCE ANALYSIS}

\subsection{Abundance Analysis Details}

We performed a standard 1D-LTE analysis using the 2017 version of the 1D LTE radiative transfer code MOOG (Sneden 1973; Sobeck et al. 2011) ${ }^{2}$ and the Castelli \& Kurucz (2004) (ATLAS) model atmospheres. We used SMHR to measure equivalent widths, interpolate model atmospheres, and run MOOG.

For the red giant branch (RGB) stars, stellar parameters were derived spectroscopically. Briefly, we start assuming $\alpha$-enhanced $[\alpha / \mathrm{Fe}]=+0.4$ model atmospheres. The effective temperature, surface gravity, and microturbulence $\left(T_{\text {eff }}, \log g, \nu_{t}\right)$ were determined by balancing excitation, ionization, and line strength for Fe lines, respectively. We then applied the $T_{\text {eff }}$ correction from

\footnotetext{
2 https://github.com/alexji/moog17scat
} 
Table 4. Line Measurements

\begin{tabular}{lrrrrrrrrrrrrrrrr}
\hline \hline Star & $\lambda$ & $\mathrm{ID}$ & $\chi$ & $\log g f$ & $\mathrm{EW}$ & $\sigma(\mathrm{EW})$ & $\mathrm{ul}$ & $\log \epsilon$ & $\sigma_{i}$ & $\sigma_{i, \mathrm{stat}}$ & $\sigma_{i, \mathrm{sys}}$ & $\delta_{i, T_{\text {eff }}}$ & $\delta_{i, \log g}$ & $\delta_{i, u_{t}}$ & $\delta_{i,[\mathrm{M} / \mathrm{H}]}$ & $\sigma_{\mathrm{cont}}$ \\
\hline CarII-0064 & 5183.60 & 12.0 & 2.72 & -0.17 & 251.8 & 7.0 & 0 & 5.21 & 0.28 & 0.04 & 0.28 & +0.23 & -0.14 & -0.08 & -0.01 & 0.07 \\
CarII-0064 & 5528.40 & 12.0 & 4.35 & -0.50 & 87.0 & 4.7 & 0 & 5.35 & 0.14 & 0.07 & 0.12 & +0.10 & -0.05 & -0.05 & -0.01 & 0.04 \\
CarII-0064 & 4323.00 & 106.0 & $\ldots$ & $\ldots$ & syn & syn & 0 & 6.16 & 0.30 & 0.03 & 0.30 & +0.28 & -0.07 & +0.01 & +0.03 & $\ldots$ \\
CarII-0064 & 4554.00 & 56.1 & 0.00 & 0.16 & syn & syn & 0 & -2.52 & 0.17 & 0.08 & 0.15 & +0.10 & +0.11 & -0.01 & +0.01 & 0.03 \\
CarII-0064 & 4129.70 & 63.1 & 0.00 & 0.22 & syn & syn & 1 & -1.89 & $\ldots$ & $\ldots$ & $\ldots$ & $\ldots$ & $\ldots$ & $\ldots$ & $\ldots$ & $\ldots$ \\
\hline
\end{tabular}

NoтE-A portion of this table is shown for form. The full version is available online. See Appendix A for column details.

Frebel et al. (2013) to place the measurements on a photometric temperature scale and redetermined $\log g$ and $\nu_{t}$. After this initial determination, if the star turned out to have low $\mathrm{Mg}$ abundances, we switched to $[\alpha / \mathrm{Fe}]=0$ atmospheres and redetermined the stellar parameters. Statistical stellar parameter uncertainties are found following Ji et al. (2019a), and we adopt systematic uncertainties of $150 \mathrm{~K}$ for $T_{\text {eff }}, 0.3 \mathrm{dex}$ for $\log g$, and $0.2 \mathrm{~km} \mathrm{~s}^{-1}$ for $\nu_{t}$ due to uncertainties in the Frebel et al. (2013) temperature calibration. The statistical and systematic uncertainties were added in quadrature to obtain the total stellar parameter uncertainties in Table 3.

We used a combination of equivalent widths and spectral syntheses to measure the abundances of individual lines. We also determined statistical and systematic abundance uncertainties for each individual feature. For lines measured using equivalent widths, we propagated the $1 \sigma$ equivalent width uncertainty into a $1 \sigma$ statistical abundance uncertainty. For lines measured using syntheses, we increased the element abundance until $\Delta \chi^{2}=1$, also corresponding to a $1 \sigma$ statistical uncertainty. These uncertainties account for continuum placement uncertainty (see Appendix A for details). For the systematic uncertainties, we varied each stellar parameter $\left(T_{\text {eff }}, \log g, \nu_{t},[\mathrm{M} / \mathrm{H}]\right)$ individually by its error and remeasured the abundance. The total systematic uncertainty is the quadrature sum of the individual stellar parameter uncertainties. Finally, the total abundance uncertainty for an individual line is the quadrature sum of the statistical and systematic uncertainty. Individual line measurements and uncertainties are found in Table 4.

We use inverse-variance weighted averages to combine lines into a final abundance. Because we have included a detailed account of line-by-line uncertainties, this automatically downweights lines in regions of low spectral $\mathrm{S} / \mathrm{N}$; saturated lines that are sensitive to small equivalent width variations; and lines that are particularly sensitive to stellar parameters. We verified that the weighted averages are usually only a few hundredths of a dex different from the unweighted averages. The exception is elements with few measurable lines like Si and $\mathrm{Al}$, where some lines are much lower quality than others. See Appendix A for detailed equations.

$[\mathrm{X} / \mathrm{Fe}]$ ratios are derived by taking ratios of common ionization states (e.g., [MgI/FeI], [TiII/FeII]). This mostly (though not always) results in smaller $[\mathrm{X} / \mathrm{Fe}]$ errors than $[\mathrm{X} / \mathrm{H}]$ errors, since some stellar parameter differences cancel out. We also consistently propagate stellar parameter uncertainties for $[\mathrm{X} / \mathrm{Y}]$ ratios, such as $[\mathrm{Mg} / \mathrm{Ca}]$.

Upper limits were derived by spectrum synthesis. For a given feature, we fit a synthetic spectrum that wellmatched the observed spectrum to determine a reference $\chi^{2}$ and local spectrum smoothing. Then holding the continuum and smoothing fixed, we increased the abundance until $\Delta \chi^{2}=25$. This is formally a $5 \sigma$ upper limit but does not include uncertainties in continuum placement.

\subsection{Abundance corrections}

Various systematics can affect 1D-LTE abundances of red giants. We tabulate several abundance corrections in Table 5, which are the average of line-by-line corrections. These corrections have been applied in all figures but not in Tables 4 or 6.

Carbon is systematically converted to nitrogen in evolved red giants due to $\mathrm{CN}$ cycling. We estimate the natal carbon abundances of these stars with the corrections from Placco et al. (2014) ${ }^{3}$. Hotter stars have no correction, while for cooler/more evolved stars the correction can be as large as +0.75 dex. We use the default correction grid assuming $[\mathrm{N} / \mathrm{Fe}]=0$, but changing $[\mathrm{N} / \mathrm{Fe}]$ makes minimal difference. Note that we assume all our stars are on the RGB, but if we had red clump or

\footnotetext{
3 http://vplacco.pythonanywhere.com/
} 
Table 5. Abundance Corrections

\begin{tabular}{lccc}
\hline \hline Star & CH Corr. & Na Corr. & Mg Corr. \\
\hline CarII-6544 & +0.75 & -0.16 & +0.03 \\
CarII-7872 & +0.60 & -0.13 & +0.03 \\
CarII-5664 & +0.74 & -0.23 & +0.05 \\
CarII-0064 & +0.61 & -0.23 & +0.04 \\
CarII-4704 & +0.62 & -0.23 & +0.03 \\
CarII-9296 & +0.49 & -0.26 & +0.04 \\
CarII-2064 & +0.01 & -0.48 & +0.03 \\
CarII-4928 & +0.01 & -0.32 & +0.03 \\
CarII-V3 & $\ldots$ & $\ldots$ & $\ldots$ \\
CarIII-1120 & +0.39 & -0.43 & +0.02 \\
CarIII-8144 & +0.01 & -0.47 & +0.02 \\
\hline
\end{tabular}

AGB stars in our sample they would have larger carbon corrections than applied here.

Only the $\mathrm{Na} \mathrm{D}$ lines are available for sodium abundances, and these can have fairly large negative NLTE corrections. We apply $\mathrm{Na}$ corrections from Lind et al. $(2011)^{4}$, which range from -0.13 to -0.48 dex. For CarII-6544 and CarII-7872, and CarII-5664 we set $\log g=1$ to avoid the edge of the corrections grid.

$\mathrm{Mg}$ is marginally affected by NLTE effects in our stars. However, since $\mathrm{Mg}$ will be a very important element later, we tabulate the NLTE corrections just to show they are only affected by $<0.04$ dex (Osorio et al. 2015; Osorio \& Barklem 2016). For several stars (CarII-6544, CarII-4704, CarII-0064, CarII-5664, CarII-7872) we set $\log g=1.5$ to avoid the edge of the corrections grid. Note that we have used the two high-equivalent width $\mathrm{Mg} \mathrm{b}$ lines in all our Mg abundances, but removing these two lines everywhere does not significantly affect our RGB star abundances.

Other elements that are known to have significant NLTE corrections include Al, Mn, K, and Fe. For these elements we do not calculate star-by-star corrections, but instead just estimate the magnitude and direction of a typical correction. If desired, the effect of these corrections can be approximated by adding the correction to the relevant abundance, as well as adding the total correction in quadrature to the total abundance error; but we do not do so here.

For aluminum, we measured the $3944 \AA$ and $3961 \AA$ lines, which are heavily affected by NLTE in cool metalpoor stars as well as being in the wings of strong lines,

4 ww. inspect-stars.com so we only estimate the abundance corrections. We examined the corrections grid from Nordlander \& Lind $(2017)^{5}$ for these lines. Half of our stars are cooler and have lower $\log g$ than the grid range. The abundance corrections for the $3961 \AA$ line tend to be large and positive, from +0.7 to +1.5 dex. The corrections for $3944 \AA$ are more moderate, from +0.0 to +0.5 dex. The corrections for these lines tend to go in opposite directions, such that averaging corrections for these lines in the warmer stars $\left(T_{\text {eff }} \gtrsim 4800 \mathrm{~K}\right)$ gives corrections in a smaller range from +0.5 to +0.7 dex. However, this also tends to make the individual $3944 \AA$ and $3961 \AA$ abundances more discrepant. Given these uncertainties, we caution against overinterpretation of our Al abundances or trends.

For manganese, we always use the resonant triplet near $4030 \AA$, as well as redder lines (e.g. $4754 \AA, 4783 \AA$ ) when detected. Bergemann et al. (2019) have recently published grids of Mn corrections, showing overall corrections of about +0.4 to +0.6 dex, though the corrections are likely larger for cooler and metal-poor stars. As our Mn abundances just fall within the overall halo trend (which are also not corrected for NLTE), we will not discuss this further.

For potassium, we can measure the $7699 \AA$ line in all stars. The $7665 \AA$ line was also clear of telluric lines for a few stars, and when measurable is always consistent with the $7699 \AA$ line. K has negative NLTE corrections that could be as large as -0.9 dex (Ivanova \& Shimanskii 2000), although Reggiani et al. (2019) have recently calculated grids of corrections that are more typically -0.0 to -0.4 dex in our stellar parameter range.

FeI abundances are affected by NLTE effects, with corrections typically +0.2 to +0.3 dex in our parameter range (e.g., Bergemann et al. 2012; Mashonkina et al. 2016; Ezzeddine et al. 2017). Our temperature correction procedure partially accounts for these effects, though not completely (Frebel et al. 2013; Ji et al. 2016b). We have decided not to apply Fe corrections so as to be able to compare our Fe measurements to literature values, which are essentially all done in LTE.

Finally, we note that $\mathrm{Ca}$ can be affected by NLTE as well (Mashonkina et al. 2016). The available grids do not span our whole stellar parameter space ${ }^{6}$, but the available corrections are about +0.1 dex for our stars. We have not applied this correction.

\subsection{RRL Abundance analysis}

\footnotetext{
5 https://www.mso.anu.edu.au/ thomasn/NLTE/

6 http://spectrum.inasan.ru/nLTE/
} 
Stellar parameters for the RRL star CarII-V3 were determined by examining the phase-parameter relations in For et al. (2011). As our observations are between phases 0.40 to 0.55 , stellar parameters are expected to be fairly stable over all exposures. We adopted initial stellar parameters of $T_{\text {eff }}=6000 \pm 100 \mathrm{~K}, \log g=$ $1.80 \pm 0.2 \mathrm{dex}, \nu_{t}=3.00 \pm 0.20 \mathrm{~km} \mathrm{~s}^{-1}$ where the error bars are adopted systematic uncertainties based on scatter in the For et al. (2011) values. Then, we measured equivalent widths by fitting Gaussian profiles to the line list from For \& Sneden (2010) (rather than our usual line list, which is optimized for red giants). To slightly improve Fe excitation, ionization, and line strength balance from $28 \mathrm{FeI}$ lines and $10 \mathrm{Fe}$ II lines, we adjusted the stellar parameters to $T_{\text {eff }}=6150 \mathrm{~K}, \log g=1.75 \mathrm{dex}$, $\nu_{t}=3.15 \mathrm{~km} \mathrm{~s}^{-1}$, resulting in $[\mathrm{M} / \mathrm{H}]=-2.70$. Total stellar parameter and abundance uncertainties were then determined the same way as the RGB stars. We do not apply any abundance corrections for this star, as the correction grids are computed for cool giants. CarII-V3 is one of the most metal-poor RRLs ever studied spectroscopically, with similar $[\mathrm{Fe} / \mathrm{H}]$ as $\mathrm{X}$ Ari and the most Fe-poor RRLs in the LMC (For et al. 2011; Haschke et al. 2012; Nemec et al. 2013).

\subsection{Abundance Summary}

Our full abundance results are tabulated in Table 6 (Appendix B) and Figures 2 and 4. We compare the results to halo stars in small grey points (Abohalima \& Frebel 2018), and to other UFD measurements in the literature. The UFD literature compilation includes Bootes I (Feltzing et al. 2009; Norris et al. 2010; Gilmore et al. 2013; Ishigaki et al. 2014; Frebel et al. 2016), Bootes II (Ji et al. 2016a), Canes Venatici II (François et al. 2016), Coma Berenices (Frebel et al. 2010), Grus I (Ji et al. 2019a), Hercules (Koch et al. 2008, 2013), Horologium I (Nagasawa et al. 2018), Leo IV (Simon et al. 2010; François et al. 2016), Pisces II (Spite et al. 2018), Reticulum II (Ji et al. 2016c; Roederer et al. 2016), Segue 1 (Frebel et al. 2014), Segue 2 (Roederer \& Kirby 2014), Triangulum II (Ji et al. 2019a; Kirby et al. 2017; Venn et al. 2017), Tucana II (Ji et al. 2016b; Chiti et al. 2018a), Tucana III (Hansen et al. 2017; Marshall et al. 2018), and Ursa Major II (Frebel et al. 2010). We reiterate that throughout this paper, the error bars for Car II and III include full propagation of the line-by-line statistical and stellar parameter uncertainties.

The RRL star CarII-V3 generally has consistent abundances with the RGB stars, although there are fewer lines and only moderate $\mathrm{S} / \mathrm{N}$ so the abundance uncertainties for this star are fairly large. The main outlier is the $\mathrm{Si}$ abundance, which is unusually low but has large uncertainty as it is measured only from the $3905 \AA$ line. Given the abundance similarities to other stars in Car II, we will treat this star's abundances on the same footing as RGB stars when lines are detected.

$C, N, O$. Carbon abundances are derived from synthesizing the $\mathrm{CH}$ bands at $\sim 4300-4325 \AA$. CO molecular equilibrium affects $\mathrm{CH}$ abundances, and we always assume the MOOG default of $[\mathrm{O} / \mathrm{Fe}]=0$ even when $\mathrm{O}$ is measured independently. Literature measurements suggest $[\mathrm{O} / \mathrm{Fe}]$ is typically $>0.5$ (e.g., Brown et al. 2014). If we used $[\mathrm{O} / \mathrm{Fe}]=+1.0$ instead, $[\mathrm{C} / \mathrm{Fe}]$ would typically increase by +0.08 dex with star-to-star scatter of 0.08 dex, but we keep the MOOG default for consistency with previously analyzed literature stars. Nitrogen is derived from fitting $\mathrm{CN}$ bands at $\sim 3850 \AA$ after fixing the $\mathrm{CH}$ abundance.

In two relatively cool and metal-rich stars, we detect the two forbidden oxygen lines at $\sim 6300 \AA$. These can only be measured when the $\mathrm{O}$ abundance is very high, so are probably a biased sample of measurements. The stronger $6300 \AA$ line was deblended from telluric absorption, and the weaker $6363 \AA$ line can be affected by a wide calcium ionization feature (e.g., Barbuy et al. 2015). However in both cases, the two different lines give very close abundances. We include oxygen upper limits for all stars (including the two detections) in the machinereadable version of Table 4 from the $6300 \AA$ line.

$\alpha$-elements: $\mathrm{Mg}, \mathrm{Si}, \mathrm{Ca}$. The $\alpha$-element abundances are determined from equivalent widths in all stars. Magnesium is determined from 5-7 lines including the $\mathrm{Mg} \mathrm{b}$ lines in all stars (except CarII-V3, where only the $\mathrm{Mg} \mathrm{b}$ lines can be measured). The $\mathrm{Mg}$ b lines are quite strong and saturated but give similar abundances as the weaker lines for all stars. Si is measured from both the $3905 \AA$ and $4102 \AA$ lines, but these are both rather poor-quality lines. The $3905 \AA$ line is fairly saturated, and the $4102 \AA$ line is in a Balmer wing. Ca is usually measured from 10-20 lines with three exceptions: the warmer and more Fe-poor stars CarII-4928 and CarIII-1120 have only 2 and 1 Ca lines, respectively; and only the strong $4226 \AA$ line is detected in the RRL CarII-V3. We do not use the $4226 \AA$ line in any of the RGB stars due to large and uncertain NLTE corrections (e.g., Sitnova et al. 2019).

Odd-Z elements: $N a, A l, K, S c$. We use equivalent widths to measure sodium abundances from the two $\mathrm{Na}$ $\mathrm{D}$ lines, which have been corrected for NLTE effects. We synthesize the $3944 \AA$ and $3961 \AA$ Al lines, which are both very strong and subject to NLTE effects so our $\mathrm{Al}$ abundances are very uncertain. $\mathrm{K}$ abundances are mostly from the $7699 \AA$ line, although occasionally the $7665 \AA$ is not blended with tellurics. Sc abundances are mostly measured with spectral synthesis from five lines 

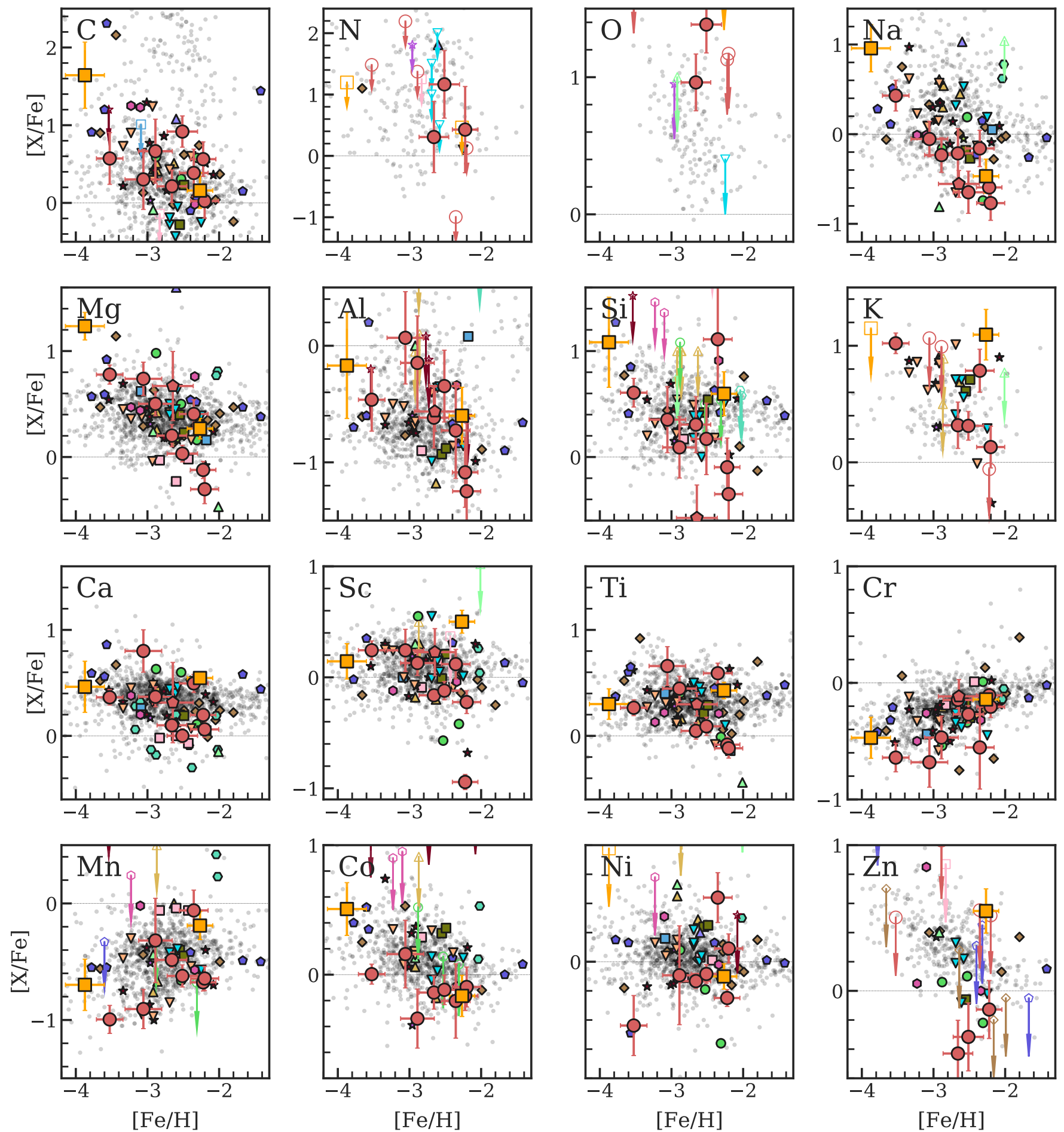

$\begin{array}{llllllllll}\diamond \text { Halo } & \bigcirc & \text { ComBer } & \square & \text { Leo IV } & \star & \text { Segue 2 } & 0 & \text { UMa II } \\ \diamond & \text { Bootes I } & \square & \text { Gru I } & \Delta & \text { Psc II } & \Delta & \text { Tri II } & \bigcirc & \text { Car II } \\ \Delta & \text { Bootes II } & \square & \text { Hercules } & \star & \text { Ret II } & \nabla & \text { Tuc II } & \square & \text { CarII-V3 } \\ \star & \text { CVn II } & \square & \text { Hor I } & \Delta & \text { Segue 1 } & \nabla & \text { Tuc III } & \square & \text { Car III }\end{array}$

Figure 2. $[\mathrm{X} / \mathrm{Fe}]$ ratios for most measured elements. Car II and Car III are shown as large red circles and large orange squares, respectively, with error bars. The RRL CarII-V3 is shown separately as a red pentagon. Other UFDs are shown as small colored points according to the legend. Upper limits are indicated by an open point with a downward-pointing arrow. The JINAbase halo sample is shown as small grey points in the background. 

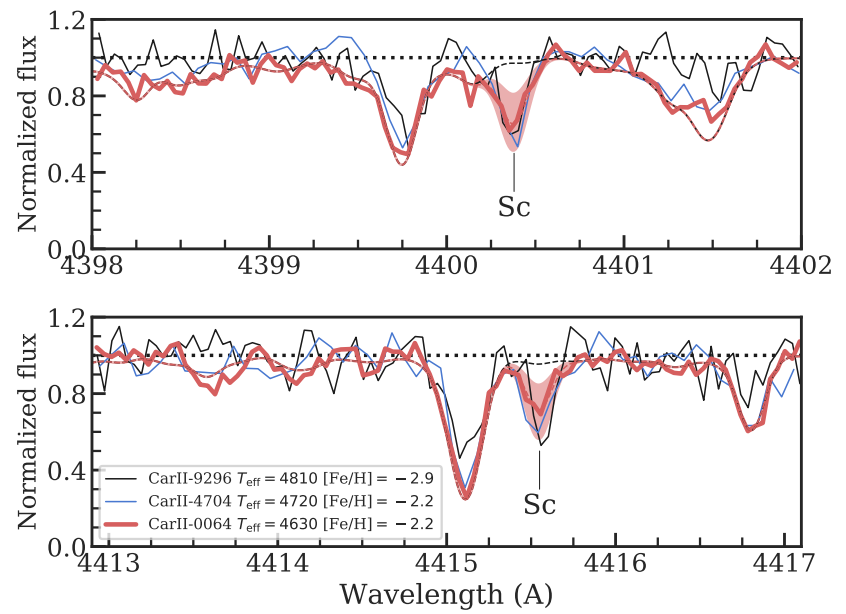

Figure 3. Spectrum of the low-Sc outlier CarII-0064 around two Sc lines, compared to two other Car II stars with similar temperature but lower Sc abundance. The Sc line is deficient in CarII-0064 compared to these other stars despite this star being somewhat cooler. Note that there is $\mathrm{C}-\mathrm{H}$ absorption in CarII-0064 near the $4400 \AA$ line. We also show the synthetic spectrum fit to the Sc line for CarII-0064 as a thin dashed red line, $\mathrm{a} \pm 0.5$ dex difference to the synthetic fit as a shaded region, and a synthesis with no Sc as a dashed black line.

at $4246<\lambda<4415 \AA$, though the redder line abundances (e.g. $5031 \AA, 5526 \AA$ ) agree.

CarII-0064 is a significant low Sc outlier in Car II with $[\mathrm{Sc} / \mathrm{Fe}] \approx-1$ (Figure 2 ). We plot two Sc line spectrum in Figure 3, along with its synthetic fit and two other stars that have higher Sc abundances. The Sc abundance is clearly lower in CarII-0064, though visually not as much as would be expected from Figure 2 . This is because each individual line difference is significant at $\lesssim 2 \sigma$, but they are all consistent and the combination of $5-6 \mathrm{Sc}$ lines reduces the uncertainty. Also note the $[\mathrm{Sc} / \mathrm{Fe}]$ abundance error is smaller, due to correlated uncertainties in stellar parameters. Such low Sc abundances have previously been seen in "iron-rich" stars (those with overall low $[\mathrm{X} / \mathrm{Fe}]$ ratios, e.g., Cohen \& Huang 2010; Cohen et al. 2013; Yong et al. 2013). However, this cannot explain CarII-0064 because it is an outlier from the overall Car II trend only in $[\mathrm{Sc} / \mathrm{Fe}]$. Similarly Sc-deficient stars have been found in the bulge where it has been argued that this signature may indicate unusually old stars (Casey \& Schlaufman 2015), but we see no sign of this in the more Fe-poor stars in Car II. It is unclear to us how to interpret this star's extreme $\mathrm{Sc}$ abundance.

Fe-peak elements: Ti, Cr, Mn, Co, Ni, Zn. We use equivalent widths to measure abundances for both ionization states of titanium, but we adopt the Ti II abundances everywhere as our default; it is measured in all
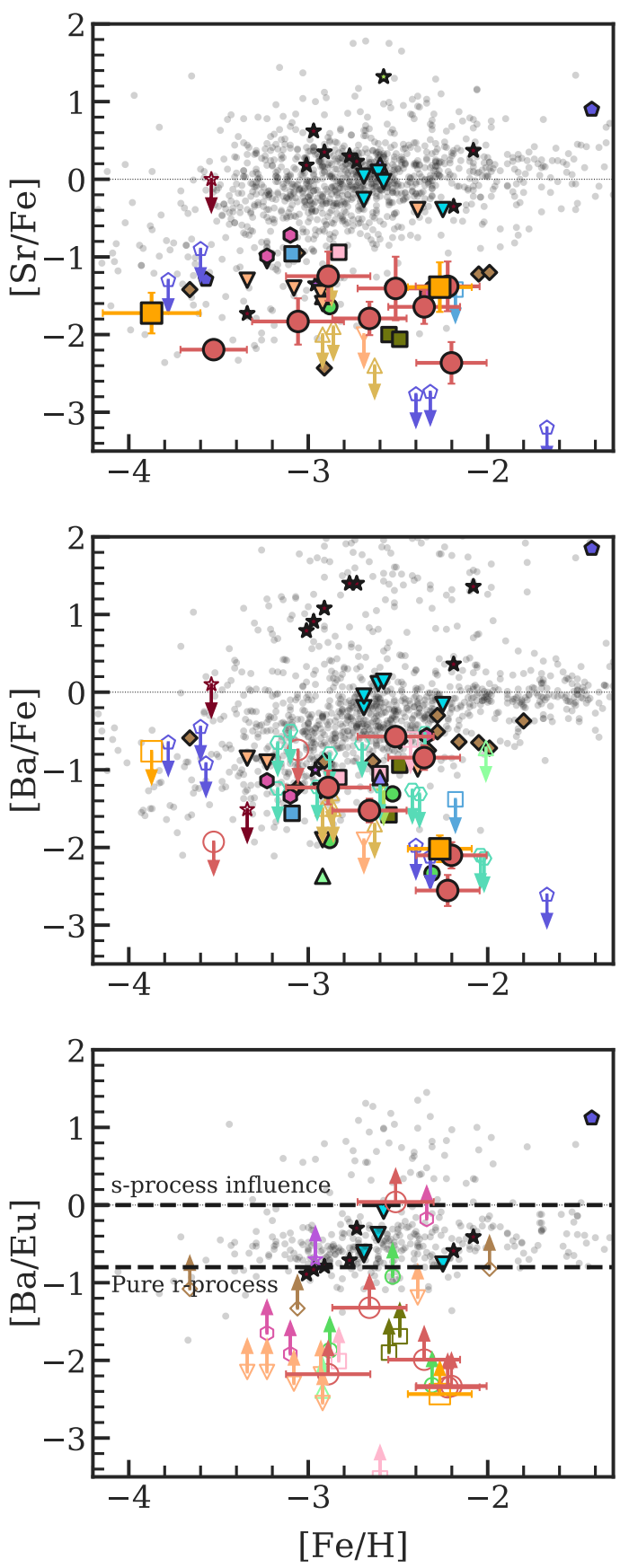

Figure 4. Neutron-capture element abundances in Car II (large red circles), Car III (large orange squares), halo stars (grey points), and other UFDs (small colored points, see Figure 2 for legend). Top and middle panels show [Sr, $\mathrm{Ba} / \mathrm{Fe}] ;$ bottom panel shows $[\mathrm{Ba} / \mathrm{Eu}]$. We draw lines at $[\mathrm{Ba} / \mathrm{Eu}]=-0.8$ and 0.0 , indicating a pure $r$-process ratio and an s-process-influenced ratio (Sneden et al. 2008) Car II and III match most other UFDs as being deficient in $\mathrm{Sr}$ and $\mathrm{Ba}$. Car II displays significant scatter in $[\mathrm{Ba} / \mathrm{Fe}]$ at $[\mathrm{Fe} / \mathrm{H}] \sim-2.5$. One Ba-rich star in Car II has $[\mathrm{Ba} / \mathrm{Eu}]>0$ and thus likely has significant $s$-process enrichment. 
our stars, has more and stronger lines, and is less susceptible to NLTE effects.

The Fe-peak elements closely follow the halo trends within their abundance uncertainties. There are minor deviations that are all significant at $<2 \sigma$, so we do not concern ourselves with these further, other than to comment that Zn could be moderately enhanced in Car III and moderately deficient in Car II.

Neutron-capture elements: Sr, Ba. These elements have low abundances or upper limits, similar to most other UFDs. The nucleosynthetic origin of these very low $\mathrm{Sr}$ and $\mathrm{Ba}$ abundances remains unknown (it is in general not even clear if they are from the $r$ - or $s$-processes, see Ji et al. (2019a) for an extensive discussion), but it appears to be unique to UFDs and occasional halo stars that are presumably stripped from UFDs. Given the low abundance of neutron-capture elements, no other neutron-capture elements could be detected, so we place $[\mathrm{Eu} / \mathrm{Fe}]$ upper limits and show $[\mathrm{Ba} / \mathrm{Eu}]$ in Figure 4.

There are two stars in Car II with relatively high $[\mathrm{Ba} / \mathrm{Fe}] \gtrsim-1$ compared to the other Car II stars. One of these relatively Ba-rich stars, CarII-7872, also has a low Eu upper limit that results in $[\mathrm{Ba} / \mathrm{Eu}] \gtrsim 0$, suggesting its $\mathrm{Ba}$ is predominantly from the $s$-process (e.g., Sneden et al. 2008). We discuss this large barium scatter in Section 4.4 .

\section{FORMATION HISTORY OF CARINA II AND III}

\subsection{Carina II and III are Dwarf Galaxies}

Low luminosity stellar systems are classified as either dwarf galaxies or star clusters. Dwarf galaxies are generally more spatially extended than clusters, with velocity dispersions implying significant dark matter content and nonzero metallicity (or more specifically, iron-peak abundance) dispersions (Willman \& Strader 2012). Faint dwarf galaxies also tend to display very low abundances of neutron-capture elements (e.g., Ji et al. 2019a), while globular clusters have light element anticorrelations associated with hot bottom burning (e.g., Bastian \& Lardo 2018).

Both Carina II and III are clearly dwarf galaxies and not globular clusters. Their half-light radii and luminosities place them within the dwarf galaxy morphological locus (Torrealba et al. 2018). Carina II displays both a significant velocity and metallicity dispersion from medium-resolution data (Li et al. 2018). Our two Carina III stars have $[\mathrm{Fe} / \mathrm{H}]$ values that differ by almost 2 dex, definitively establishing a significant metallicity dispersion. We have also now resolved the velocity dispersion ( $\mathrm{Li}$ et al. in prep). The neutron-capture ele- ments $\mathrm{Sr}$ and $\mathrm{Ba}$ are low in both systems, like nearly every other UFD (Figure 4).

These criteria alone already show that Car II and III are galaxies, but as a final confirmation we show there are no light element anticorrelations. Figure 5 shows these relations for our stars. In the top two panels, we show Na-Mg and Al-Mg for our UFD stars (symbols as in Figure 2) and globular cluster stars as purple circles (from references Carretta et al. 2007, 2009; Gratton et al. 2006; Cohen \& Kirby 2012). Most globular clusters do not show significant dispersion in $[\mathrm{Mg} / \mathrm{Fe}]$, but those that do always display an anti-correlation in $\mathrm{Na}-\mathrm{Mg}$ and $\mathrm{Al}-\mathrm{Mg}$. In contrast, there is very clearly a positive correlation for these elements in both Car II and III. Note that $\mathrm{Na}$ and $\mathrm{Mg}$ have NLTE corrections applied, while the $\mathrm{Al}$ corrections should on average provide an offset and are unlikely to turn a strong positive $\mathrm{Mg}$ - $\mathrm{Al}$ correlation into an anticorrelation. The bottom panel of Figure 5 shows the $\mathrm{Mg}-\mathrm{K}$ anticorrelation found in NGC 2419 (Mucciarelli et al. 2012), which is not present in Car II. However, our two stars in Car III (including one $\mathrm{K}$ upper limit) do not rule out an $\mathrm{Mg}-\mathrm{K}$ anticorrelation in this system.

\subsection{Car II and III are consistent with being accreted along with the $L M C / S M C$}

Li et al. (2018) showed that the positions and radial velocities of both Car II and Car III were consistent with having accreted with the LMC, according to the Jethwa et al. (2016) model. Kallivayalil et al. (2018) then added proper motion data from Gaia DR2 (Gaia Collaboration et al. 2018, 2016), finding that Car II and Car III are also likely LMC satellites based on LMC analogues in the Aquarius simulations (Springel et al. 2008, also see Sales et al. 2017; Simon 2018; Erkal \& Belokurov 2019). Kinematically, it thus appears likely that both Car II and Car III entered the Milky Way with the LMC/SMC system, although Car II is towards the edge of the likely region due to its high velocity. Kallivayalil et al. (2018) also associate Hyi I and Hor I with the LMC.

Thus, Car II and III, along with Hor I (Nagasawa et al. 2018), can be studied in contrast to other UFDs to see if abundance ratios have any environmental dependence. Nagasawa et al. (2018) point out that the three stars in Hor I have unusually low $\mathrm{Mg}$ and $\mathrm{Ca}$, with one possible explanation being that LMC satellites might have typically different enrichment histories compared to Milky Way UFDs. Figure 2 does not suggest that Car II or Car III obviously deviate from the typical abundance scatter of other UFDs, including for $\mathrm{Mg}$ and $\mathrm{Ca}$. The unusually low $\mathrm{Mg}$ and $\mathrm{Ca}$ in Hor I thus likely has some other origin. 

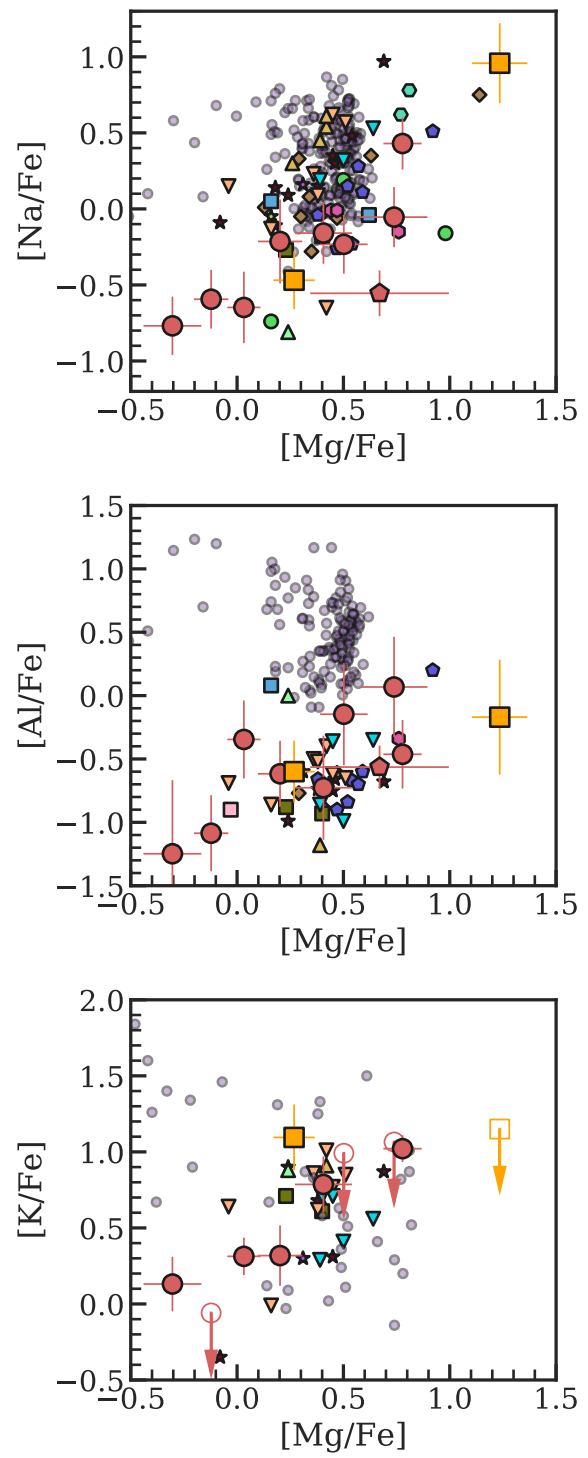

Figure 5. $\mathrm{Mg}-\mathrm{Na}, \mathrm{Mg}-\mathrm{Al}$, and $\mathrm{Mg}-\mathrm{K}$ abundance patterns. Car II is red circles/pentagon, Car III is orange squares, globular cluster stars are small purple circles, and other UFDs are small colored points (same as Figure 2). In globular clusters, $\mathrm{Mg}-\mathrm{Na}$ and $\mathrm{Mg}-\mathrm{Al}$ are anti-correlated, while in both Car II and Car III these elements are clearly correlated. $\mathrm{Mg}-\mathrm{K}$ are anticorrelated in the globular cluster NGC 2419, and there is no evidence for such in Car II. The light element correlations confirm that Car II and III are dwarf galaxies and not globular clusters.

\section{3. $\alpha$-element evolution: time delay scenario or initial mass function variations?}

\subsection{1. $\alpha$-element abundance ratios in Car II and Car III}

The $\alpha$-elements $(\mathrm{O}, \mathrm{Mg}, \mathrm{Si}, \mathrm{Ca})$ are primarily produced in core-collapse supernovae (CCSNe) and thus tend to be enhanced at low $[\mathrm{Fe} / \mathrm{H}]$. After a delay of 100 - 1000 Myr (Maoz et al. 2014), Type Ia super- novae (SNe1a) begin to add Fe peak elements, causing a "knee" in $[\alpha / \mathrm{Fe}]$ vs $[\mathrm{Fe} / \mathrm{H}]$ (Tinsley 1979). In this time delay scenario, the location of the knee can be interpreted as an overall star formation timescale for a galaxy (e.g., Tolstoy et al. 2009; Kirby et al. 2011). Figure 2 shows clear downward trends in $[\mathrm{Mg} / \mathrm{Fe}]$ and $[\mathrm{Ca} / \mathrm{Fe}]$ vs. $[\mathrm{Fe} / \mathrm{H}]$ for both Car II and III, with a possible knee at $[\mathrm{Fe} / \mathrm{H}] \sim-2.8$ for Car II that would indicate very slow chemical evolution in this low mass galaxy.

However, there is a striking difference in the size of the trend for $[\mathrm{Mg} / \mathrm{Fe}]$ and $[\mathrm{Ca} / \mathrm{Fe}]:[\mathrm{Mg} / \mathrm{Fe}]$ declines by over 1 dex, while $[\mathrm{Ca} / \mathrm{Fe}]$ declines by only about 0.4 dex. We will focus primarily on Car II, because Car III has only two stars and the more Fe-poor star has only one Ca line. To clarify the $\mathrm{Mg}$ and $\mathrm{Ca}$ difference, in the top panel of Figure 6 we plot $[\mathrm{Mg} / \mathrm{Ca}]$ vs $[\mathrm{Fe} / \mathrm{H}]$, where $[\mathrm{Mg} / \mathrm{Ca}]$ declines from about +0.4 to -0.4 as $[\mathrm{Fe} / \mathrm{H}]$ increases from -3.5 to -2.2 . These extreme $[\mathrm{Mg} / \mathrm{Ca}]$ ratios are often interpreted as variations in the high mass end of the initial mass function. Stars with high $[\mathrm{Mg} / \mathrm{Ca}]$ ratios are typically associated with enrichment by very massive stars with $M>20-30 M_{\odot}$ (e.g., Norris et al. 2000; Cohen et al. 2007; Koch et al. 2008, also see Section 5.2). Stars with $[\mathrm{Mg} / \mathrm{Ca}]<0$ form out of gas enriched by lower mass CCSN progenitors with $M \lesssim 15 M_{\odot}$ (e.g., Tolstoy et al. 2003; McWilliam et al. 2013). The variable $[\mathrm{Mg} / \mathrm{Ca}]$ ratios in Car II may thus indicate that the $\alpha$ elements in this galaxy is tracing changes in the highmass end of the initial mass function (IMF). Indeed, the low-mass end of the IMF in UFDs has previously been shown to vary between different UFDs (Geha et al. 2013; Gennaro et al. 2018), which tantalizingly hints that the high-mass end of the IMF might vary as well (although the low-mass IMF varies from galaxy to galaxy, while here we consider time variations within a single galaxy, so the mechanisms may not be related).

In the bottom panels of Figure 6, we plot $[\mathrm{Mg} / \mathrm{H}]$ and $[\mathrm{Ca} / \mathrm{H}]$ vs $[\mathrm{Fe} / \mathrm{H}]$, which shows that there may actually be two phases of $[\mathrm{Mg} / \mathrm{Ca}]$ evolution: from $[\mathrm{Fe} / \mathrm{H}]=-3.6$ to -3.0 this is primarily driven by a smaller increase in $[\mathrm{Mg} / \mathrm{H}]$ than $[\mathrm{Ca} / \mathrm{H}]$; while from $[\mathrm{Fe} / \mathrm{H}]=-3.0$ to -2.2 , $[\mathrm{Mg} / \mathrm{H}]$ stays mostly flat while $[\mathrm{Ca} / \mathrm{H}]$ increases. The first phase unambiguously shows that Car II has been enriched by at least two different masses of CCSNe: the most Fe-poor star in Car II has high $[\mathrm{Mg} / \mathrm{Ca}]$ ratios suggesting enrichment by high mass stars, but it has lower $[\mathrm{Mg} / \mathrm{H}]$ than the higher metallicity stars. Since SNe1a produce negligible $\mathrm{Mg}$, this means that $\mathrm{CCSNe}$ with $[\mathrm{Mg} / \mathrm{Ca}] \sim 0$ must have enriched Car II after the formation of the most Fe-poor star. This could potentially be evidence of a transition from very massive Pop III stars to regular mass Pop II CCSNe. 

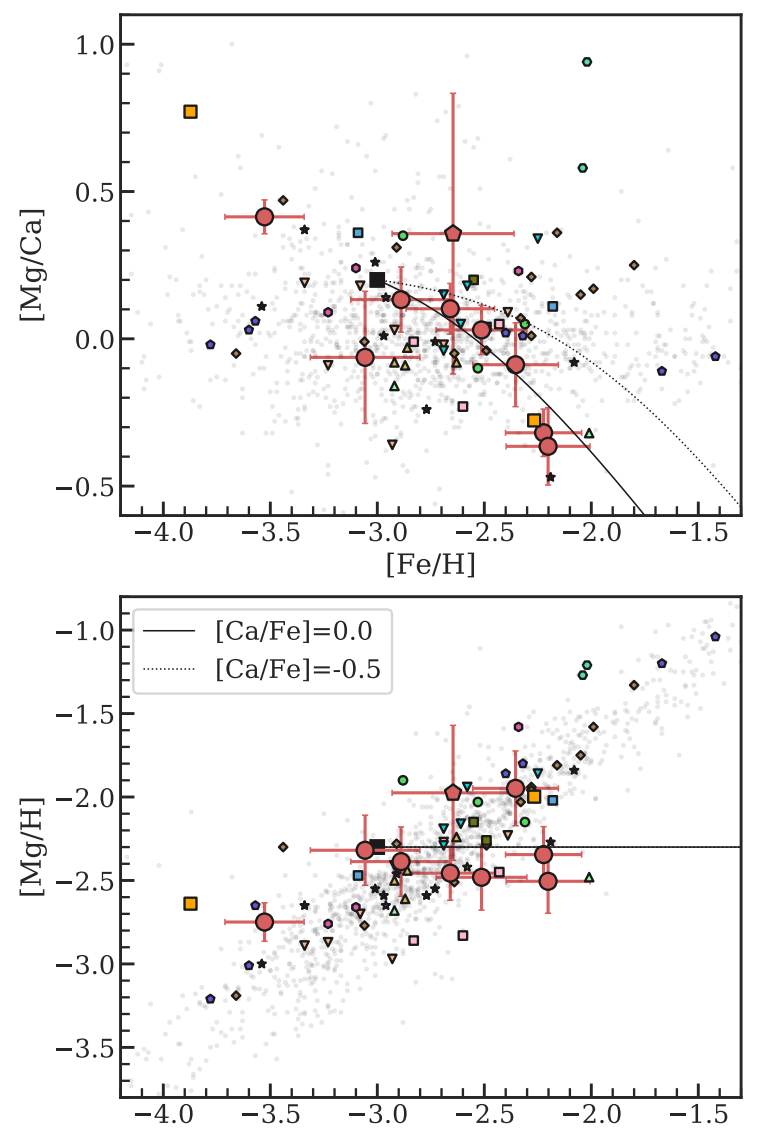

$[\mathrm{Fe} / \mathrm{H}]$

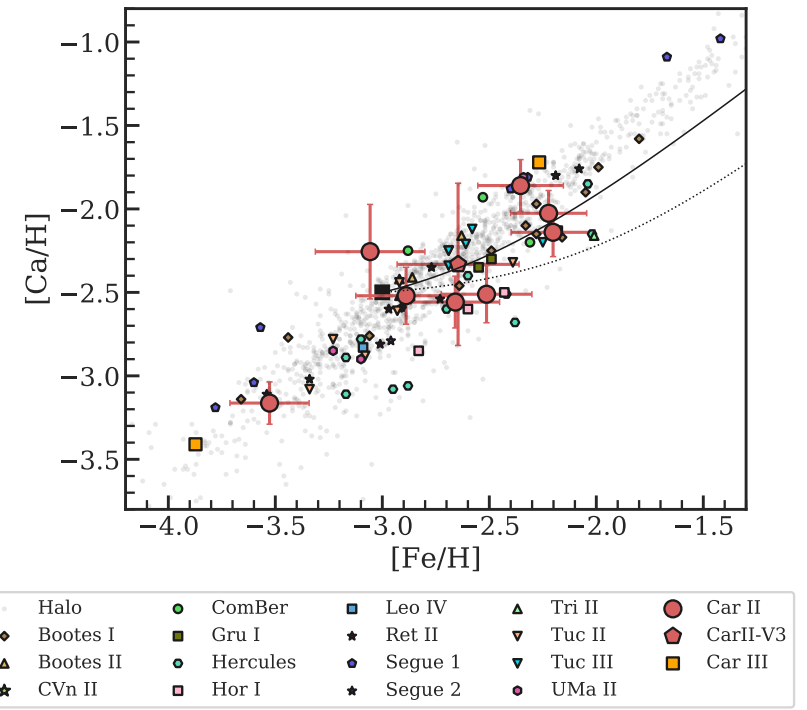

Figure 6. Top panel: $[\mathrm{Mg} / \mathrm{Ca}]$ vs $[\mathrm{Fe} / \mathrm{H}]$ for UFDs (colored points) and halo stars (small grey points). We focus here particularly on Car II (large red points with error bars). Middle and bottom panels: $[\mathrm{Mg} / \mathrm{H}]$ and $[\mathrm{Ca} / \mathrm{H}]$ as a function of metallicity. On all panels, the solid and dotted black lines show tracks of SN1a-only enrichment for two SN1a Ca yields, starting at the black square.
The second phase of evolution could be attributed to either IMF variation or SN1a enrichment. To illustrate this, we show an extremely simple chemical evolution track in Figure 6. First, we set an initial $[\mathrm{Mg} / \mathrm{H}]$, $[\mathrm{Ca} / \mathrm{H}]$, and $[\mathrm{Fe} / \mathrm{H}]$ that matches the $[\mathrm{Mg} / \mathrm{Ca}]$ ratio at $[\mathrm{Fe} / \mathrm{H}]=-3$ (black square). Then, we assume a fixed $[\mathrm{Ca} / \mathrm{Fe}]$ yield and negligible $\mathrm{Mg}$ yield for SNe1a (Kirby et al. 2019), and compute the evolution of $\mathrm{Mg}$, Ca, and $\mathrm{Fe}$ assuming no more $\mathrm{CCSNe}$ and no gas accretion/expulsion. Kirby et al. (2019) have recently made an empirical measurement of the $\mathrm{SN1} 1 \mathrm{a}[\mathrm{Ca} / \mathrm{Fe}]$ yield in larger $\mathrm{dSph}$ galaxies, finding values that range between $-0.5<[\mathrm{Ca} / \mathrm{Fe}]<0.0$. We thus apply our simple model with SN1a yields of $[\mathrm{Ca} / \mathrm{Fe}]=0.0$ and -0.5 , which are shown as black solid and dotted lines respectively in Figure 6 and reasonably match the observed $\mathrm{Mg}$ and Ca ratios. This would be quite an extreme situation: if most of the metal enrichment in Car II is due to SNe1a and not CCSNe, but stars still formed to sample the SN1a yields, that implies an extremely top-light IMF where no massive stars formed. However, this is definitely not a unique model, and specifically the flat $[\mathrm{Mg} / \mathrm{H}]$ trend does not rule out contributions from additional CCSNe because gas accretion can increase the hydrogen reservoir (e.g., Ji et al. 2016a). Detailed chemical evolution modeling of more elements might help clarify the picture but is beyond the scope of this paper. Furthermore, stochastic sampling of individual SN explosions may dominate the observed trends (e.g., Koch et al. 2008, 2013; Revaz et al. 2016; Applebaum et al. 2018), especially given that Car II produced only $\sim 100$ CCSNe in total (assuming a Salpeter initial mass function and present-day mass-to-light ratio of 2.2 , Ji et al. 2016a). Car III is even more susceptible to stochastic enrichment, having been enriched by only $\sim 15$ supernovae. We thus caution against over-interpreting the available data.

\subsection{2. $[\mathrm{Mg} / \mathrm{Ca}]$ abundances across the UFD population}

Some more insight can be derived by comparing the $[\mathrm{Mg} / \mathrm{Ca}]$ vs $[\mathrm{Fe} / \mathrm{H}]$ trends of Car II to the trends in other UFDs. It turns out that few other UFDs have similarly negative $[\mathrm{Mg} / \mathrm{Ca}]$ vs $[\mathrm{Fe} / \mathrm{H}]$ slopes. To quantify this result, we fit lines to the $[\mathrm{Mg} / \mathrm{Ca}]$ vs $[\mathrm{Fe} / \mathrm{H}]$ evolution of every UFD individually, and consider the slope angle (i.e., $0^{\circ}$ corresponds to a flat line, and negative slope angles indicate declining $[\mathrm{Mg} / \mathrm{Ca}]$ as $[\mathrm{Fe} / \mathrm{H}]$ increases). We then calculate the slopes and slope uncertainties by assuming that data points are drawn from a thin line with multivariate Gaussian uncertainties (see section 7 of Hogg et al. 2010). We take a uniform prior in slope angle (as opposed to slope) for $\theta \in\left[-90^{\circ},+90^{\circ}\right.$ ) and a 

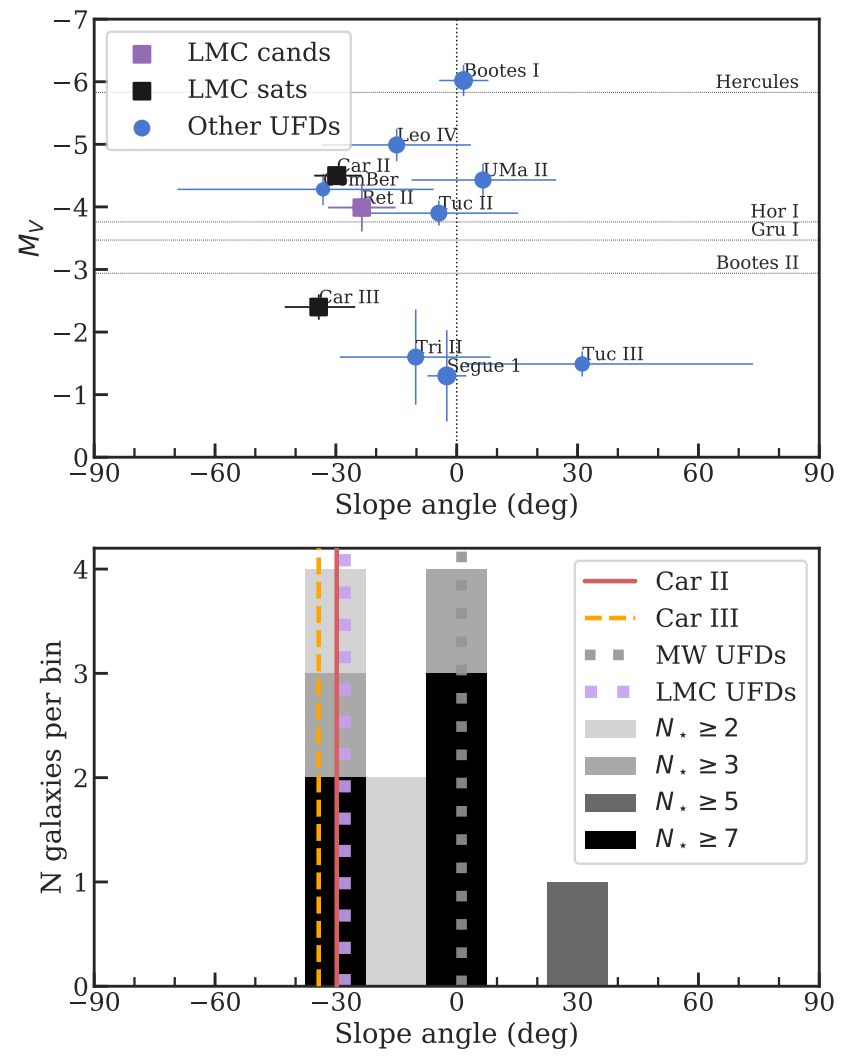

Figure 7. Top: $[\mathrm{Mg} / \mathrm{Ca}]$ vs $[\mathrm{Fe} / \mathrm{H}]$ slope angles for all UFDs vs luminosity $M_{V}$. Slope error bars indicate $68 \%$ posterior region, and measurements with smaller uncertainties have correspondingly larger symbols. The luminosities of galaxies with unconstrained slope posteriors are shown as horizontal lines. Square symbols indicate UFDs that are LMC satellites (Car II, Car III, Hor I) or satellite candidates (Ret II). Round symbols indicate UFDs likely associated with the Milky Way. Bottom: histogram of $[\mathrm{Mg} / \mathrm{Ca}]$ slope angles for all UFDs. UFDs with more stars (i.e., more confident slope measurements) are shown as darker shades of grey. The relatively extreme slope angles for Car II and III are marked as vertical solid and dashed lines, respectively. The total $[\mathrm{Mg} / \mathrm{Ca}]$ slopes for all MW vs LMC UFD stars are marked in dotted grey and purple lines, respectively.

flat prior for the intercept, then use emcee to sample the posterior (Foreman-Mackey et al. 2013). We take the posterior median as the point estimate and the 16th84 th percentile range as the $68 \%$ credible interval. We remove the four UFDs that have unconstrained posteriors (since their stars have essentially the same $[\mathrm{Fe} / \mathrm{H}]$ ). Note that the literature UFD stars have inhomogenously determined uncertainties, so we instead assume independent error bars of 0.2 dex for both $[\mathrm{Fe} / \mathrm{H}]$ and $[\mathrm{Mg} / \mathrm{Ca}]$; but use our actual abundance uncertainties for Car II and Car III.

The $[\mathrm{Mg} / \mathrm{Ca}]$ vs $[\mathrm{Fe} / \mathrm{H}]$ slopes for all UFDs where $\geq 2$ stars have detailed abundance measurements are shown in Figure 7. The top panel of Figure 7 shows the UFD $[\mathrm{Mg} / \mathrm{Ca}]$ slopes vs luminosity (luminosities from the Simon 2019 compilation, including data from Bechtol et al. 2015; Muñoz et al. 2018; Torrealba et al. 2018; MutluPakdil et al. 2018). There is not an obvious relation between slope angle and luminosity. The bottom panel shows a histogram of the slope angle point estimates from the top panel. Many UFDs have too few stars to place a useful slope constraint, so we shade each UFD in the histogram by the number of stars used to calculate the slope, with darker colors indicating more stars. The UFDs with the most confident measurements (i.e., $\geq 7$ stars with detailed abundances) are Car II (this work), Ret II (Ji et al. 2016c), Bootes I (Frebel et al. 2016), Segue 1 (Frebel et al. 2014), and Tuc II (Chiti et al. 2018a). We also highlight the slope of Car II and Car III as a vertical solid red line and vertical orange dashed line, respectively. Of the other UFDs, only Ret II exhibits a declining $[\mathrm{Mg} / \mathrm{Ca}]$ slope that deviates from zero by $\gtrsim 1 \sigma$.

\subsubsection{Effect of environment on $[\mathrm{Mg} / \mathrm{Ca}]$ abundances}

The results above raise an interesting question about the role of environment in determining abundance trends: Car II and III are LMC satellites, and Ret II is also a candidate LMC satellite (Kallivayalil et al. 2018; Erkal \& Belokurov 2019) ${ }^{7}$. In the bottom panel of Figure 7 , we show the $[\mathrm{Mg} / \mathrm{Ca}]$ vs $[\mathrm{Fe} / \mathrm{H}]$ slope angles from grouping all LMC UFD stars and all MW UFD stars. It is very obvious that the LMC satellite UFD stars have a significant negative slope, while the MW satellite UFD stars have a flat slope; though we note that the LMC trend is mostly driven by Car II and should await additional abundances in LMC satellite UFDs to clarify this suggestion.

However, we speculate briefly on how the large scale environment could possibly affect chemical evolution in UFDs. At first glance, UFDs should not display significant environment dependence. UFDs form most of their stars by $z \sim 6$ (Brown et al. 2014), and in simulations the closest more massive galaxy at $z>6$ is typically 400 physical kpc away (Wetzel et al. 2015). Even generously sized galactic superbubbles reach only tens of kpc (Griffen et al. 2018), so external enrichment or directly affecting UFD gas with ram pressure stripping is unlikely (Wetzel et al. 2015). However, radiation (both ionizing and Lyman-Werner) can span these dis-

\footnotetext{
${ }^{7}$ Hor I (Nagasawa et al. 2018) also is an LMC satellite, but all three currently observed stars have $[\mathrm{Fe} / \mathrm{H}] \sim-2.6$ within uncertainties and thus no useful constraint on its $[\mathrm{Mg} / \mathrm{Ca}]$ vs $[\mathrm{Fe} / \mathrm{H}]$ trend. The three Hor I stars all have $[\mathrm{Mg} / \mathrm{Fe}] \approx[\mathrm{Ca} / \mathrm{Fe}] \approx 0$.
} 
tances, though there are limited ways we can imagine this would affect stellar populations. At the metal-rich end, one possibility is the integrated galactic IMF theory (IGIMF, e.g., Weidner et al. 2013; McWilliam et al. 2013), which suggests that as galaxies become gas-poor they cannot form the most massive stars. If LMC UFDs formed later and thus reionized earlier in their evolution, they would form more of their stars in this phase. At the metal-poor end, delaying Pop III star formation with Lyman-Werner feedback may increase susceptibility of UFD progenitors to external enrichment (e.g., Magg et al. 2018). Also, metal-free gas with relatively high ionization fractions can form HD molecules during collapse, which may (or may not) affect the Pop III initial mass function (Glover 2013). A final note is that the distance scales from Wetzel et al. (2015) assume that UFDs reside in dark matter halos of $M_{\text {peak }} \sim 10^{9} M_{\odot}$ (Wetzel et al. 2015). If instead UFDs reside in smaller dark matter halos of $M_{\text {peak }} \sim 10^{7-8} M_{\odot}$ (e.g., Jeon et al. 2014; Ji et al. 2015; Jethwa et al. 2018; Graus et al. 2019), then separation distances would become smaller and environmental effects could be more important.

\subsection{Inhomogenous metal mixing of $A G B$ winds in Car II}

There is real scatter in $[\mathrm{Ba} / \mathrm{Fe}]$ at $[\mathrm{Fe} / \mathrm{H}] \sim-2.5$ in Car II, with some stars having relatively high Ba abundances and others having low Ba abundances (Figure 4). The extent of the scatter in $\mathrm{Ba}$ is $\sim 1$ dex, much larger than the scatter in any other abundance ratio. A plausible explanation for the Ba scatter is inhomogeneous mixing of AGB wind ejecta into the galaxy's ISM. Unlike supernova ejecta, which mix rapidly upon entering the hot phase of the ISM, AGB winds mix into relatively cool ISM phases and can thus stay quite inhomogeneous (Emerick et al. 2018, 2019). Since Ba is produced by the $s$-process and released in AGB winds, this mechanism could explain the large $\mathrm{Ba}$ scatter. This scenario is supported by the fact that one of the high-Ba stars (CarII-7872) has [Ba/Eu] $\gtrsim 0$ (Figure 4), suggesting its $\mathrm{Ba}$ is predominantly from the $s$-process. Since most barium comes from AGB stars with initial mass $M \leq 4 M_{\odot}$ and lifetimes $\geq 10^{8}$ years, the presence of AGB enrichment requires that Car II formed stars for at least $\sim 100$ Myr (Lugaro et al. 2012; Karakas \& Lugaro 2016). Note that the nucleosynthetic origin of the low $\mathrm{Sr}$ and $\mathrm{Ba}$ floor in UFDs remains unknown (see Ji et al. 2019a, for more discussion). One might also expect a correlation between $\mathrm{Ba}$ and other AGB elements like $\mathrm{C}$. We find a moderate but not statistically significant correlation between stars that have both $\mathrm{Ba}$ and $\mathrm{C}$ detected in
Car II (correlation of 0.48 with a $p$-value of 0.34 from scipy.stats.pearsonr).

\section{POPULATION III STAR SIGNATURES}

\subsection{Carbon-enhanced fraction in UFDs}

Carbon-Enhanced Metal-Poor (CEMP) stars are stars with high $[\mathrm{C} / \mathrm{Fe}]$ ratios (Beers \& Christlieb 2005). Below $[\mathrm{Fe} / \mathrm{H}] \sim-3$, about half the stars in the Milky Way halo are CEMP stars (i.e., [C/Fe] $\gtrsim+0.7$, Aoki et al. 2007). It is generally thought that a specific subclass (CEMPno stars; Beers \& Christlieb 2005) ${ }^{8}$ of the CEMP stars traces unique nucleosynthesis in Pop III stars (e.g., Norris et al. 2013; Frebel \& Norris 2015; Placco et al. 2016). If so, the observed CEMP fraction provides a window to the distribution of some Pop III star properties, such as initial mass, explosion energy, or stellar rotation (e.g., Cooke \& Madau 2014; Ji et al. 2015).

In Figure 8, we show the fraction of carbon-enhanced stars below a given $[\mathrm{Fe} / \mathrm{H}]$ in our $\gtrsim 80$ star UFD literature sample and the halo star compilation by Placco et al. (2014). Both samples have included the Placco et al. (2014) evolutionary carbon corrections. For the UFD sample, we show $68 \%$ Wilson confidence intervals on the CEMP fraction. Figure 8 shows that the carbon-enhanced fraction in UFDs is essentially identical to halo stars at all levels of carbon enhancement. For comparison, the CEMP fraction in larger dwarf galaxies like Sculptor has been studied in some detail (e.g., Skúladóttir et al. 2015; Salvadori et al. 2015; Chiti et al. 2018 b), but it is still debated whether the CEMP fraction in those galaxies is consistent with the halo.

If we are after pure Pop III signatures, it also makes sense to look at entire UFDs as either C-rich or C-normal (Ji et al. 2015). Seven UFDs have stars with $[\mathrm{Fe} / \mathrm{H}]<$ -3 . The most metal-poor stars in five of these UFDs are C-rich (Car III, Segue 1, Boo I, Tuc II, UMa II), while the other two are C-normal (Ret II, Car II). This suggests that the fraction of Pop III stars producing carbon-enhanced abundances is $0.71_{-0.19}^{+0.13}$, following the simple models in Ji et al. (2015). A more stringent cut of $[\mathrm{Fe} / \mathrm{H}]<-3.5$ results in three C-enhanced galaxies out of five, or a carbon-enhanced rate of $0.60_{-0.39}^{+0.34}$. More to the point, the existence of carbon-normal stars with $[\mathrm{Fe} / \mathrm{H}] \lesssim-3.5$ in Ret II and Car II is evidence against the hypothesis that $100 \%$ of Pop III stars produce carbon-enhanced signatures, as is often assumed in theoretical models and simulations (e.g., Salvadori et al. 2015; Jeon et al. 2017).

\footnotetext{
8 The " $n o$ " is short for "no strong enhancement of neutroncapture elements".
} 


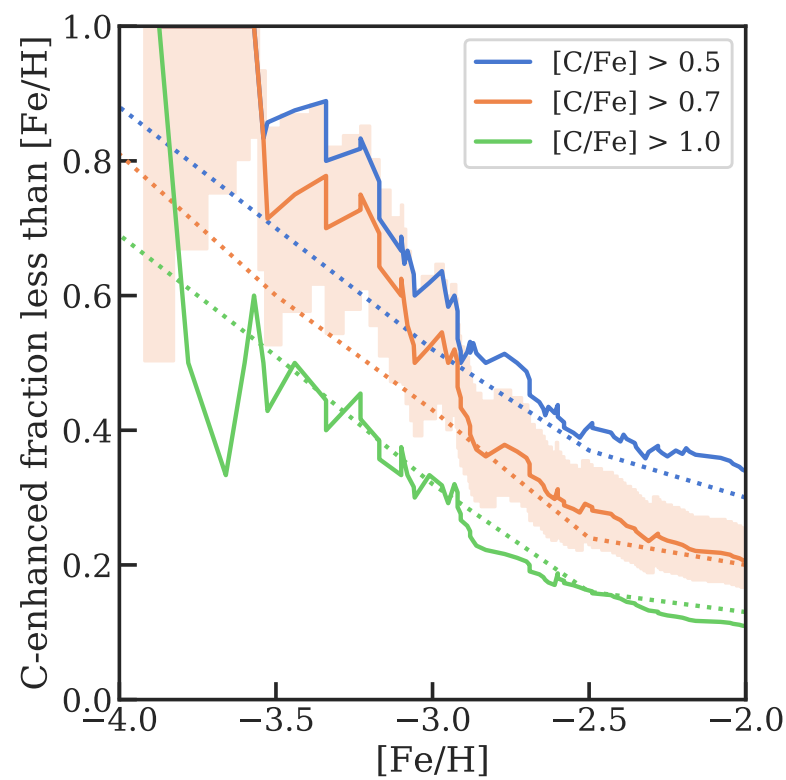

Figure 8. Cumulative carbon-enhanced metal-poor (CEMP) fraction. The solid colored lines show the CEMP fraction for UFD stars at different C-enhanced cutoffs, with the shaded region indicating the $68 \%$ Wilson confidence interval for a binomial distribution around the $[\mathrm{C} / \mathrm{Fe}]>0.7$ fraction. The dotted colored lines show the halo CEMP fraction from Placco et al. (2014). Both the UFD data and the reference sample have included carbon evolutionary corrections. The UFD CEMP fraction is consistent with the halo.

\subsection{Full fits to individual UFD stars}

The two stars CarII-5664 and CarIII-1120 have low enough $[\mathrm{Fe} / \mathrm{H}]$ that they are plausibly enriched only by Pop III stars (e.g., Frebel \& Norris 2015). Under this assumption, we fit models from Heger \& Woosley (2010) to the data to estimate the initial progenitor mass, explosion energy, internal mixing, and gas dilution mass for these stars. To summarize the fitting procedure, we find the optimum dilution mass for all 16800 models in the Heger \& Woosley (2010) grid, reject all models inconsistent with our upper limits, then weight each remaining model by using its deviation from the best-fit $\chi^{2}$ as input to a $\chi^{2}$ survival function with 4 degrees of freedom. The detailed fitting procedure and parameter description is described in Frebel et al. $(2019)^{9}$. Here, we exclude the elements $\mathrm{Al}, \mathrm{K}$, and $\mathrm{Mn}$ due to the uncertain size of NLTE corrections; and the elements $\mathrm{Sc}, \mathrm{Cr}, \mathrm{Cu}$, and $\mathrm{Zn}$ due to model calculation uncertainties (Heger \& Woosley 2010). Abundance corrections to C, Na, and

9 Code at https://github.com/alexji/alexmods/blob/ master/alexmods/alex_starfit.py
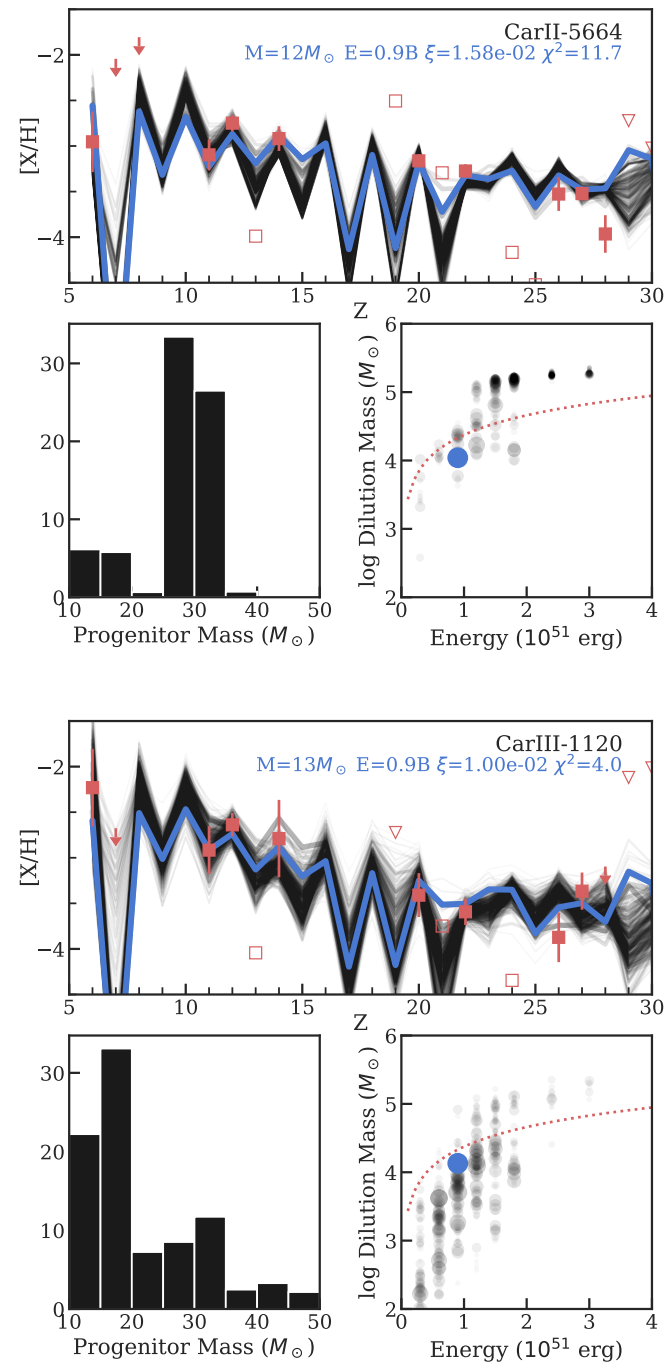

Figure 9. Pop III SN yield fits to abundances of the three stars with $[\mathrm{Fe} / \mathrm{H}] \lesssim-3.5$. Top panels: the measured abundances (red), the single best-fit model (blue) and all models within $2 \sigma$ (black). Bottom left panels: weighted histogram of the best-fit progenitor masses. Bottom right panels: the model energy and dilution masses. The best fit model is shown as a blue point. The dashed red line indicates the minimum dilution mass for a given energy. See text for details.

$\mathrm{Mg}$ have been included (Table 5). We note that the Heger \& Woosley (2010) models do not include stellar rotation. However, rotation can substantially influence stellar evolution and the resulting nucleosynthesis (e.g., Maeder et al. 2015) and should be considered in future analyses.

The results are shown in Figure 9. We plot all models within $2 \sigma$ contours of $\chi^{2}$ (i.e., models with weight $\gtrsim 0.05)$. In the top panel for each star, we show the data as filled red squares with error bars and upper limits as downward pointing arrows. Unused measurements and 
upper limits are indicated as open squares and downward pointing triangles, respectively. The best-fit model is shown as a solid blue line, while other models within $2 \sigma$ are shown as black lines. For visualization purposes, models with worse $\chi^{2}$ are plotted as thinner transparent lines. The bottom left panel for each star shows the weighted histogram for the resulting progenitor masses of the full fit. The bottom right panel shows the bestfit energy and dilution masses, where again models with worse $\chi^{2}$ are displayed as smaller and more transparent points. The best fit model is again shown as a solid blue point. In general, satisfactory fits were found for these two stars with $[\mathrm{Fe} / \mathrm{H}]<-3.5$. CarIII-1120 is most consistent with a relatively low mass progenitor between $10-20 M_{\odot}$ with a typical $\sim 1 \times 10^{51}$ erg explosion energy. Note that CarIII-1120 is a Group 2 CEMP-no star according to Yoon et al. (2016). CarII-5664 is also best fit by a similar low-mass progenitor, but most of the best-fit models actually prefer a higher mass progenitor of $25-35 M_{\odot}$ with slightly higher explosion energy.

The combination of explosion energy and dilution mass introduces another consistency check. A supernova with explosion energy $E$ will produce a supernova remnant that sweeps up a certain amount of mass before merging with the ISM (e.g., Cioffi et al. 1988). This is the minimum dilution mass allowable for that explosion energy (assuming no rare interactions such as colliding supernova blastwaves). In the bottom right panels of Figure 9 we show the approximate swept-up mass of a supernova remnant expanding into an efficiently cooling ISM $M_{\text {dil, } \mathrm{H}}=0.75 \times 10^{4.5} M_{\odot}\left(E / 10^{51} \mathrm{erg}\right)^{0.95}$ as a dotted red line (Cioffi et al. 1988; Ryan et al. 1996). Models below this line are inconsistent with the explosion energy (though could be explained with enrichment by multiple supernovae), while models above the line are diluted beyond the supernova remnant due to turbulent mixing. Applying this constraint tends to prefer higher explosion energies and higher masses. In general, the best-fit dilution masses satisfying this constraint are $\sim 10^{5} M_{\odot}$, suggesting that recollapsed gas within a minihalo is the most likely explanation for the origin of these stars rather than external pollution, as externally polluted halos have higher effective dilution masses (e.g., Cooke \& Madau 2014; Ji et al. 2015; Smith et al. 2015; Griffen et al. 2018).

\section{CONCLUSION}

We present a comprehensive abundance analysis of the Magellanic satellite galaxies Carina II and Carina III using high-resolution Magellan/MIKE data, including the first abundances of an RR Lyrae star in any UFD. The abundance results are shown in Figures 2 and 4.
The stars in these two dwarf galaxies clearly do not show light element anticorrelations associated with globular clusters (Figure 5).

The most notable chemical evolution trend is the variations in different $\alpha$-element ratios. Car II clearly shows different trends in $[\mathrm{Mg} / \mathrm{Fe}]$ and $[\mathrm{Ca} / \mathrm{Fe}]$ (Figure 6). The origin of this evolution could be differences in core collapse and/or Type Ia supernova yields, and it is not yet clear which. However, there are obvious differences in the $[\mathrm{Mg} / \mathrm{Ca}]$ trends between different UFDs (Figure 7), and we tentatively suggest this could be an environmentdependent abundance signature as LMC satellite UFDs have a different trend than MW satellite UFDs. This suggestion will require studying the abundances of additional LMC satellites to confirm.

The most metal-poor stars in UFDs may contain signatures of the first metal-free Population III stars. Studying the whole population of Fe-poor UFD stars, we find that the carbon-enhanced fraction of UFD stars is essentially the same as the Milky Way halo (Figure 8). But, not all of the most Fe-poor stars in UFDs are carbon-enhanced: the most Fe-poor star in Car II is clearly carbon-normal. We also found two new stars with $[\mathrm{Fe} / \mathrm{H}] \leq-3.5$, bringing the total number of such stars in UFDs up to 8. The abundances of these stars are well-fit by Pop III core-collapse supernova yields (Figure 9).

Our analysis of Car II and III, along with the past decade of observations, brings the total number of UFD stars with high-resolution abundances up to $\sim 85$ stars across 16 different UFDs, of which now 5 UFDs have a "large" $(\geq 7)$ number of stars studied (see references in Section 3.4). While these data have already provided key insights into early nucleosynthesis and galaxy formation and pointed to many interesting abundance trends and signatures, the numbers of stars are still relatively small. These sample sizes are currently dictated by the limits of current large telescopes, but $30 \mathrm{~m}$ class telescopes will allow high-resolution spectroscopic abundances for $10 \mathrm{~s}-100 \mathrm{~s}$ of stars per UFD out to the virial radius of the Milky Way (Ji et al. 2019b), transforming our ability to unravel the detailed history of these first galaxy relics.

We thank Andy McWilliam, Evan Kirby, Ian Thompson, George Preston, Dan Kelson, Andrew Emerick, and Thomas Nordlander, and Chris Sneden for fruitful discussions; and Eduardo Bañados for saving our RRL observations from certain doom. APJ and TSL are supported by NASA through Hubble Fellowship grant HST-HF2-51393.001 and HST-HF2-51439.001 respectively, awarded by the Space Telescope Science In- 
stitute, which is operated by the Association of Universities for Research in Astronomy, Inc., for NASA, under contract NAS5-26555. JDS is supported by the National Science Foundation under grant AST-1714873. SK is partially supported by NSF awards AST-1813881 and AST-1909584. The work of ABP is supported by NSF grant AST-1813881. This research has made use of NASA's Astrophysics Data System Bibliographic Services.

This work has made use of data from the European Space Agency (ESA) mission Gaia (https : //www . cosmos.esa.int/gaia), processed by the Gaia Data Processing and Analysis Consortium (DPAC, https:// www . cosmos . esa.int/web/gaia/dpac/consortium). Funding for the DPAC has been provided by national institutions, in particular the institutions participating in the Gaia Multilateral Agreement.

This project used data obtained with the Dark Energy Camera (DECam), which was constructed by the Dark Energy Survey (DES) collaboration. Funding for the DES Projects has been provided by the U.S. Department of Energy, the U.S. National Science Foundation, the Ministry of Science and Education of Spain, the Science and Technology Facilities Council of the United Kingdom, the Higher Education Funding Council for England, the National Center for Supercomputing Applications at the University of Illinois at UrbanaChampaign, the Kavli Institute of Cosmological Physics at the University of Chicago, the Center for Cosmology and Astro-Particle Physics at the Ohio State University, the Mitchell Institute for Fundamental Physics and Astronomy at Texas A\&M University, Financiadora de Estudos e Projetos, Fundação Carlos Chagas Filho de Amparo à Pesquisa do Estado do Rio de Janeiro, Conselho Nacional de Desenvolvimento Científico e Tecnológico and the Ministério da Ciência, Tecnologia e
Inovacão, the Deutsche Forschungsgemeinschaft, and the Collaborating Institutions in the Dark Energy Survey. The Collaborating Institutions are Argonne National Laboratory, the University of California at Santa Cruz, the University of Cambridge, Centro de Investigaciones Enérgeticas, Medioambientales y TecnológicasMadrid, the University of Chicago, University College London, the DES-Brazil Consortium, the University of Edinburgh, the Eidgenössische Technische Hochschule (ETH) Zürich, Fermi National Accelerator Laboratory, the University of Illinois at Urbana-Champaign, the Institut de Ciències de l'Espai (IEEC/CSIC), the Institut de Física d'Altes Energies, Lawrence Berkeley National Laboratory, the Ludwig-Maximilians Universität München and the associated Excellence Cluster Universe, the University of Michigan, the National Optical Astronomy Observatory, the University of Nottingham, the Ohio State University, the OzDES Membership Consortium the University of Pennsylvania, the University of Portsmouth, SLAC National Accelerator Laboratory, Stanford University, the University of Sussex, and Texas A\&M University.

Based on observations at Cerro Tololo Inter-American Observatory, National Optical Astronomy Observatory (NOAO Prop. ID 2016A-0366 and PI Keith Bechtol), which is operated by the Association of Universities for Research in Astronomy (AURA) under a cooperative agreement with the National Science Foundation. This project is partially supported by the NASA Fermi Guest Investigator Program Cycle 9 No. 91201.

Facilities: Magellan-Clay (MIKE)

Software: MOOG (Sneden 1973; Sobeck et al. 2011), SMHR (Casey 2014), numpy (van der Walt et al. 2011), scipy (Jones et al. 2001), matplotlib (Hunter 2007), pandas (Mckinney 2010), seaborn, (Waskom et al. 2016), astropy (Astropy Collaboration et al. 2013)

\section{REFERENCES}

Abohalima, A., \& Frebel, A. 2018, ApJS, 238, 36

Aoki, W., Beers, T. C., Christlieb, N., et al. 2007, ApJ, 655,492

Applebaum, E., Brooks, A. M., Quinn, T. R., \& Christensen, C. R. 2018, arXiv e-prints, arXiv:1811.00022

Astropy Collaboration, Robitaille, T. P., Tollerud, E. J., et al. 2013, A\&A, 558, A33

Barbuy, B., Friaça, A. C. S., da Silveira, C. R., et al. 2015, A\&A, 580, A40

Bastian, N., \& Lardo, C. 2018, ARA\&A, 56, 83

Battaglia, G., Irwin, M., Tolstoy, E., et al. 2008, MNRAS, 383,183
Bechtol, K., Drlica-Wagner, A., Balbinot, E., et al. 2015, ApJ, 807, 50

Beers, T. C., \& Christlieb, N. 2005, ARA\&A, 43, 531

Belokurov, V., Zucker, D. B., Evans, N. W., et al. 2007, ApJ, 654, 897

Benson, A. J., Frenk, C. S., Lacey, C. G., Baugh, C. M., \& Cole, S. 2002, MNRAS, 333, 177

Bergemann, M., Lind, K., Collet, R., Magic, Z., \& Asplund, M. 2012, MNRAS, 427, 27

Bergemann, M., Gallagher, A. J., Eitner, P., et al. 2019, arXiv e-prints, arXiv:1905.05200 
Bernstein, R., Shectman, S. A., Gunnels, S. M., Mochnacki, S., \& Athey, A. E. 2003, Proc. SPIE, 4841, 1694

Besla, G., Kallivayalil, N., Hernquist, L., et al. 2007, ApJ, 668, 949

Brown, T. M., Tumlinson, J., Geha, M., et al. 2014, ApJ, 796, 91

Bullock, J. S., Kravtsov, A. V., \& Weinberg, D. H. 2000, ApJ, 539, 517

Busha, M. T., Wechsler, R. H., Behroozi, P. S., et al. 2011, ApJ, 743, 117

Carretta, E., Bragaglia, A., Gratton, R., \& Lucatello, S. 2009, A\&A, 505, 139

Carretta, E., Bragaglia, A., Gratton, R. G., et al. 2007, A\&A, 464, 967

Casey, A. R. 2014, ArXiv e-prints, arXiv:1405.5968

Casey, A. R., \& Schlaufman, K. C. 2015, ApJ, 809, 110

Castelli, F., \& Kurucz, R. L. 2004, ArXiv Astrophysics e-prints, astro-ph/0405087

Chiti, A., Frebel, A., Ji, A. P., et al. 2018a, ApJ, 857, 74

Chiti, A., Simon, J. D., Frebel, A., et al. 2018b, ApJ, 856, 142

Cioffi, D. F., McKee, C. F., \& Bertschinger, E. 1988, ApJ, 334,252

Clementini, G., Ripepi, V., Molinaro, R., et al. 2019, A\&A, 622, A60

Cohen, J. G., Christlieb, N., Thompson, I., et al. 2013, ApJ, 778, 56

Cohen, J. G., \& Huang, W. 2010, ApJ, 719, 931

Cohen, J. G., \& Kirby, E. N. 2012, ApJ, 760, 86

Cohen, J. G., McWilliam, A., Christlieb, N., et al. 2007, ApJL, 659, L161

Cooke, R. J., \& Madau, P. 2014, ApJ, 791, 116

D’Onghia, E., \& Lake, G. 2008, ApJL, 686, L61

Dooley, G. A., Peter, A. H. G., Carlin, J. L., et al. 2017, MNRAS, 472, 1060

Drlica-Wagner, A., Bechtol, K., Rykoff, E. S., et al. 2015, ApJ, 813, 109

Drlica-Wagner, A., Bechtol, K., Allam, S., et al. 2016, ApJL, 833, L5

Emerick, A., Bryan, G. L., \& Mac Low, M.-M. 2019, arXiv e-prints, arXiv:1909.04695

Emerick, A., Bryan, G. L., Mac Low, M.-M., et al. 2018, ApJ, 869, 94

Erkal, D., \& Belokurov, V. A. 2019, arXiv e-prints, arXiv:1907.09484

Ezzeddine, R., Frebel, A., \& Plez, B. 2017, ApJ, 847, 142

Feltzing, S., Eriksson, K., Kleyna, J., \& Wilkinson, M. I. 2009, A\&A, 508, L1

Flaugher, B., Diehl, H. T., Honscheid, K., et al. 2015, AJ, 150,150
For, B.-Q., \& Sneden, C. 2010, AJ, 140, 1694

For, B.-Q., Sneden, C., \& Preston, G. W. 2011, ApJS, 197, 29

Foreman-Mackey, D., Hogg, D. W., Lang, D., \& Goodman, J. 2013, PASP, 125, 306

François, P., Monaco, L., Bonifacio, P., et al. 2016, A\&A, 588, A7

Frebel, A., \& Bromm, V. 2012, ApJ, 759, 115

Frebel, A., Casey, A. R., Jacobson, H. R., \& Yu, Q. 2013, ApJ, 769, 57

Frebel, A., Christlieb, N., Norris, J. E., Aoki, W., \& Asplund, M. 2006, ApJL, 638, L17

Frebel, A., Ji, A. P., Ezzeddine, R., et al. 2019, ApJ, 871, 146

Frebel, A., \& Norris, J. E. 2015, ARA\&A, 53, 631

Frebel, A., Norris, J. E., Gilmore, G., \& Wyse, R. F. G. 2016, ApJ, 826, 110

Frebel, A., Simon, J. D., Geha, M., \& Willman, B. 2010, ApJ, 708, 560

Frebel, A., Simon, J. D., \& Kirby, E. N. 2014, ApJ, 786, 74

Fritz, T. K., Carrera, R., Battaglia, G., \& Taibi, S. 2019, A\&A, 623, A129

Gaia Collaboration, Prusti, T., de Bruijne, J. H. J., et al. 2016, A\&A, 595, A1

Gaia Collaboration, Brown, A. G. A., Vallenari, A., et al. 2018, A\&A, 616, A1

Geha, M., Brown, T. M., Tumlinson, J., et al. 2013, ApJ, 771,29

Gennaro, M., Geha, M., Tchernyshyov, K., et al. 2018, ApJ, 863, 38

Gilmore, G., Norris, J. E., Monaco, L., et al. 2013, ApJ, 763, 61

Glover, S. 2013, in Astrophysics and Space Science Library, Vol. 396, The First Galaxies, ed. T. Wiklind,

B. Mobasher, \& V. Bromm, 103

Gratton, R. G., Lucatello, S., Bragaglia, A., et al. 2006, A\&A, 455, 271

Graus, A. S., Bullock, J. S., Kelley, T., et al. 2019, Monthly Notices of the Royal Astronomical Society, 488, 4585

Griffen, B. F., Dooley, G. A., Ji, A. P., et al. 2018, MNRAS, 474, 443

Hansen, T. T., Simon, J. D., Marshall, J. L., et al. 2017, ApJ, 838, 44

Haschke, R., Grebel, E. K., Frebel, A., et al. 2012, AJ, 144, 88

Heger, A., \& Woosley, S. E. 2010, ApJ, 724, 341

Hogg, D. W., Bovy, J., \& Lang, D. 2010, arXiv e-prints, arXiv:1008.4686 
Hunter, J. D. 2007, Computing in Science \& Engineering, 9, 90. http://scitation.aip.org/content/aip/journal/ cise/9/3/10.1109/MCSE. 2007.55

Ishigaki, M. N., Aoki, W., Arimoto, N., \& Okamoto, S. 2014, A\&A, 562, A146

Ivanova, D. V., \& Shimanskï, V. V. 2000, Astronomy Reports, 44, 376

Jeon, M., Besla, G., \& Bromm, V. 2017, ApJ, 848, 85

Jeon, M., Pawlik, A. H., Bromm, V., \& Milosavljević, M. 2014, MNRAS, 444, 3288

Jethwa, P., Erkal, D., \& Belokurov, V. 2016, MNRAS, 461, 2212

一. 2018, MNRAS, 473, 2060

Ji, A. P., Frebel, A., \& Bromm, V. 2015, MNRAS, 454, 659

Ji, A. P., Frebel, A., Chiti, A., \& Simon, J. D. 2016a, Nature, 531, 610

Ji, A. P., Frebel, A., Ezzeddine, R., \& Casey, A. R. 2016b, ApJL, 832, L3

Ji, A. P., Frebel, A., Simon, J. D., \& Chiti, A. 2016c, ApJ, 830, 93

Ji, A. P., Simon, J. D., Frebel, A., Venn, K. A., \& Hansen, T. T. 2019a, ApJ, 870, 83

Ji, A. P., Beaton, R., Chakrabarti, S., et al. 2019b, arXiv e-prints, arXiv:1903.09275

Jones, E., Oliphant, T., Peterson, P., et al. 2001, SciPy: Open source scientific tools for Python, , . http://www.scipy . org/

Kallivayalil, N., van der Marel, R. P., Besla, G., Anderson, J., \& Alcock, C. 2013, ApJ, 764, 161

Kallivayalil, N., Sales, L. V., Zivick, P., et al. 2018, ApJ, 867,19

Karakas, A. I., \& Lugaro, M. 2016, ApJ, 825, 26

Kelson, D. D. 2003, PASP, 115, 688

Kirby, E. N., Cohen, J. G., Simon, J. D., et al. 2017, ApJ, 838,83

Kirby, E. N., Cohen, J. G., Smith, G. H., et al. 2011, ApJ, 727, 79

Kirby, E. N., Simon, J. D., Geha, M., Guhathakurta, P., \& Frebel, A. 2008, ApJL, 685, L43

Kirby, E. N., Xie, J. L., Guo, R., et al. 2019, ApJ, 881, 45 Koch, A., Feltzing, S., Adén, D., \& Matteucci, F. 2013, A\&A, 554, A5

Koch, A., McWilliam, A., Grebel, E. K., Zucker, D. B., \& Belokurov, V. 2008, ApJL, 688, L13

Koposov, S. E., Belokurov, V., Torrealba, G., \& Evans, N. W. 2015a, ApJ, 805, 130

Koposov, S. E., Casey, A. R., Belokurov, V., et al. 2015b, ApJ, 811, 62

Laevens, B. P. M., Martin, N. F., Ibata, R. A., et al. 2015, ApJL, 802, L18
Li, T. S., Ji, A. P., \& MagLiteS Collaboration. in prep, in prep

Li, T. S., Simon, J. D., Pace, A. B., et al. 2018, ApJ, 857, 145

Lind, K., Asplund, M., Barklem, P. S., \& Belyaev, A. K. 2011, A\&A, 528, A103

Lugaro, M., Karakas, A. I., Stancliffe, R. J., \& Rijs, C. 2012, ApJ, 747, 2

Maeder, A., Meynet, G., \& Chiappini, C. 2015, A\&A, 576, A56

Magg, M., Hartwig, T., Agarwal, B., et al. 2018, MNRAS, 473, 5308

Maoz, D., Mannucci, F., \& Nelemans, G. 2014, Annual Review of Astronomy and Astrophysics, 52, 107

Marshall, J. L., Hansen, T., Simon, J. D., et al. 2018, arXiv e-prints, arXiv:1812.01022

Mashonkina, L. I., Sitnova, T. N., \& Pakhomov, Y. V. 2016, Astronomy Letters, 42, 606

Mckinney, W. 2010

McWilliam, A., Wallerstein, G., \& Mottini, M. 2013, ApJ, 778,149

Muñoz, R. R., Côté, P., Santana, F. A., et al. 2018, ApJ, 860,66

Mucciarelli, A., Bellazzini, M., Ibata, R., et al. 2012, MNRAS, 426, 2889

Mutlu-Pakdil, B., Sand, D. J., Carlin, J. L., et al. 2018, ApJ, 863, 25

Nagasawa, D. Q., Marshall, J. L., Li, T. S., et al. 2018, ApJ, 852, 99

Nemec, J. M., Cohen, J. G., Ripepi, V., et al. 2013, ApJ, 773,181

Nordlander, T., \& Lind, K. 2017, A\&A, 607, A75

Norris, J. E., Beers, T. C., \& Ryan, S. G. 2000, ApJ, 540, 456

Norris, J. E., Wyse, R. F. G., Gilmore, G., et al. 2010, ApJ, 723,1632

Norris, J. E., Yong, D., Bessell, M. S., et al. 2013, ApJ, 762, 28

Osorio, Y., \& Barklem, P. S. 2016, A\&A, 586, A120

Osorio, Y., Barklem, P. S., Lind, K., et al. 2015, A\&A, 579, A53

Pace, A. B., \& Li, T. S. 2019, ApJ, 875, 77

Placco, V. M., Frebel, A., Beers, T. C., \& Stancliffe, R. J. 2014, ApJ, 797, 21

Placco, V. M., Frebel, A., Beers, T. C., et al. 2016, ApJ, 833,21

Reggiani, H., Amarsi, A. M., Lind, K., et al. 2019, A\&A, 627, A177

Revaz, Y., Arnaudon, A., Nichols, M., Bonvin, V., \& Jablonka, P. 2016, A\&A, 588, A21 
Roederer, I. U., \& Kirby, E. N. 2014, MNRAS, 440, 2665

Roederer, I. U., Mateo, M., Bailey, III, J. I., et al. 2016, AJ, 151, 82

Ryan, S. G., Norris, J. E., \& Beers, T. C. 1996, ApJ, 471, 254

Sales, L. V., Navarro, J. F., Kallivayalil, N., \& Frenk, C. S. 2017, MNRAS, 465, 1879

Salvadori, S., Skuladottir, A., \& Tolstoy, E. 2015, ArXiv e-prints, arXiv:1506.03451

Simon, J. D. 2018, ApJ, 863, 89

-. 2019, ARA\&A, 57, 375

Simon, J. D., Frebel, A., McWilliam, A., Kirby, E. N., \& Thompson, I. B. 2010, ApJ, 716, 446

Simon, J. D., \& Geha, M. 2007, ApJ, 670, 313

Sitnova, T. M., Mashonkina, L. I., Ezzeddine, R., \& Frebel, A. 2019, MNRAS, 485, 3527

Skúladóttir, Á., Tolstoy, E., Salvadori, S., et al. 2015, A\&A, 574, A129

Smith, B. D., Wise, J. H., O'Shea, B. W., Norman, M. L., \& Khochfar, S. 2015, MNRAS, 452, 2822

Sneden, C., Cowan, J. J., \& Gallino, R. 2008, ARA\&A, 46, 241

Sneden, C. A. 1973, PhD thesis, The University of Texas at Austin.

Sobeck, J. S., Kraft, R. P., Sneden, C., et al. 2011, AJ, 141, 175

Spite, M., Spite, F., François, P., et al. 2018, A\&A, 617, A56

Springel, V., Wang, J., Vogelsberger, M., et al. 2008, MNRAS, 391, 1685

Tinsley, B. M. 1979, ApJ, 229, 1046
Tolstoy, E., Hill, V., \& Tosi, M. 2009, ARA\&A, 47, 371

Tolstoy, E., Venn, K. A., Shetrone, M., et al. 2003, AJ, 125, 707

Torrealba, G., Belokurov, V., Koposov, S. E., et al. 2018, MNRAS, 475, 5085

van der Walt, S., Colbert, S. C., \& Varoquaux, G. 2011, Computing in Science \& Engineering, 13, 22.

http://scitation.aip.org/content/aip/journal/ cise/13/2/10.1109/MCSE. 2011.37

Venn, K. A., Starkenburg, E., Malo, L., Martin, N., \& Laevens, B. P. M. 2017, MNRAS, 466, 3741

Waskom, M., Botvinnik, O., O'Kane, D., et al. 2016, seaborn: v0.7.0 (January 2016), , doi:10.5281/zenodo.45133.

http://dx.doi.org/10.5281/zenodo.45133

Weidner, C., Kroupa, P., Pflamm-Altenburg, J., \& Vazdekis, A. 2013, MNRAS, 436, 3309

Weisz, D. R., Johnson, B. D., \& Conroy, C. 2014, ApJL, 794, L3

Wetzel, A. R., Deason, A. J., \& Garrison-Kimmel, S. 2015, The Astrophysical Journal, 807, 49

Willman, B., \& Strader, J. 2012, AJ, 144, 76

Willman, B., Blanton, M. R., West, A. A., et al. 2005, AJ, 129,2692

Wise, J. H., Demchenko, V. G., Halicek, M. T., et al. 2014, MNRAS, 442, 2560

Yong, D., Norris, J. E., Bessell, M. S., et al. 2013, ApJ, 762, 26

Yoon, J., Beers, T. C., Placco, V. M., et al. 2016, ArXiv e-prints, arXiv:1607.06336 


\section{APPENDIX}

\section{A. ABUNDANCE ERROR ANALYSIS FORMALISM}

Here we explicitly list the equations used for our error analysis. For element $X$, with lines indexed by $i$ that have abundances $A_{i}$, statistical error $\sigma_{i \text {,stat }}$, and systematic abundance offsets $\delta_{i, T_{\text {eff }}}, \delta_{i, \log g}, \delta_{i, \nu_{t}}$ and $\delta_{i,[\mathrm{M} / \mathrm{H}]}$ (note that the systematic abundance offsets retain their sign so we refer to them as $\delta_{i}$ ):

$$
\begin{aligned}
\sigma_{i, \mathrm{sys}}^{2} & =\delta_{i, T_{\mathrm{eff}}}^{2}+\delta_{i, \log g}^{2}+\delta_{i, \nu_{t}}^{2}+\delta_{i,[\mathrm{M} / \mathrm{H}]}^{2} \\
& \equiv \sum_{S P} \delta_{i, S P}^{2} \\
\sigma_{i}^{2} & =\sigma_{i, \mathrm{stat}}^{2}+\sigma_{i, \mathrm{sys}}^{2}
\end{aligned}
$$

The statistical error $\sigma_{i, \text { stat }}$ quantifies the spectrum noise, either through the $1 \sigma$ equivalent width uncertainty or $\chi^{2}$ uncertainty for synthesis. Our equivalent width and synthesis fits allow the local continuum to vary by a linear function, using $\chi^{2}$ minimization to find the continuum level. Our quoted statistical uncertainties $\sigma_{i \text {,stat propagate }}$ these continuum uncertainties, and they match those inferred from simpler formulas based on the line FWHM within $5 \%$ (e.g., Battaglia et al. 2008; Frebel et al. 2006).

It is in principle possible that our local spectrum models are not accurate, and the most impactful systematic would be misplacing the overall continuum level. As an extra conservative error bar, we include an additional column $\sigma_{\text {cont }}$ in Table 4 , which is the uncertainty from systematically changing the overall continuum by the local $1 \sigma$ spectrum noise (i.e., the abundance difference after multiplying each equivalent width by $1 \pm 1 / \mathrm{SNR}$ ). For synthesis measurements, we estimate this uncertainty by calculating the equivalent width of the synthetic feature without any other elements, then treating it as an equivalent width measurement. We thus did not estimate the continuum error for the molecular features. A very conservative error estimate would also add this error in quadrature as part of equation A3. However, we are confident that our continuum placement procedure uncertainties are accurately reflected in the statistical error bar, so we do not include $\sigma_{\text {cont }}$ in our abundance uncertainties.

We then assign each line a weight $w_{i}$

$$
w_{i}=\sigma_{i}^{-2}
$$

We adopt the weighted average of the lines as the final abundance, with statistical and systematic uncertainties:

$$
\begin{aligned}
A(X) & =\frac{\sum_{i} w_{i} A_{i}}{\sum_{i} w_{i}} \\
\sigma_{\text {stat }}^{2}(X) & =\frac{\sum_{i} w_{i}\left(A_{i}-A(X)\right)^{2}}{\sum_{i} w_{i}}+\frac{1}{\sum_{i} w_{i}} \\
\delta_{\text {sys }, S P}(X) & =\frac{\sum_{i} w_{i}\left(A_{i}+\delta_{i, S P}\right)}{\sum_{i} w_{i}}-A(X) \\
& =\frac{\sum_{i} w_{i} \delta_{i, S P}}{\sum_{i} w_{i}}
\end{aligned}
$$

The total statistical uncertainty accounts for both noise in individual lines as well as the weighted standard error of different lines. Here we adopt just the first order Taylor expansion for the stellar parameter uncertainty, neglecting covariance between stellar parameters (see McWilliam et al. 2013). Finally, the total abundance error for $[\mathrm{X} / \mathrm{H}]$ and element ratios $[\mathrm{X} / \mathrm{Y}]$ combines the statistical and systematic uncertainties in quadrature:

$$
\begin{aligned}
\sigma_{[\mathrm{X} / \mathrm{H}]}^{2} & =\sigma_{\mathrm{stat}}^{2}+\sum_{S P} \delta_{\mathrm{sys}, S P}^{2} \\
\sigma_{[\mathrm{X} / \mathrm{Y}]}^{2} & =\sigma_{X, \mathrm{stat}}^{2}+\sigma_{Y, \text { stat }}^{2}+\sum_{S P}\left(\delta_{X, S P}-\delta_{Y, S P}\right)^{2}
\end{aligned}
$$

Note that for an element ratio of $\mathrm{X}$ and $\mathrm{Y}$, we only allow covariance between $X$ and $Y$ through the stellar parameters. 


\section{B. ABUNDANCE TABLES}

Table 6. Stellar Abundances

\begin{tabular}{|c|c|c|c|c|c|c|c|c|}
\hline El. & $N$ & & $\log \epsilon$ & $\sigma_{\text {stat }}$ & {$[\mathrm{X} / \mathrm{H}]$} & $\sigma_{[\mathrm{X} / \mathrm{H}]}$ & {$[\mathrm{X} / \mathrm{Fe}]$} & $\sigma_{[\mathrm{X} / \mathrm{Fe}]}$ \\
\hline \multicolumn{9}{|c|}{ CarII-0064 } \\
\hline $\mathrm{Na} \mathrm{I}$ & 2 & & 3.65 & 0.15 & -2.59 & 0.31 & -0.36 & 0.19 \\
\hline $\mathrm{Mg} \mathrm{I}$ & 5 & & 5.21 & 0.07 & -2.39 & 0.17 & -0.17 & 0.08 \\
\hline $\mathrm{Al} \mathrm{I}$ & 2 & & 3.14 & 0.23 & -3.31 & 0.30 & -1.09 & 0.30 \\
\hline Si I & 2 & & 5.19 & 0.27 & -2.32 & 0.36 & -0.10 & 0.28 \\
\hline $\mathrm{Ca} \mathrm{I}$ & 22 & & 4.31 & 0.03 & -2.03 & 0.14 & 0.20 & 0.06 \\
\hline Sc II & 6 & & 0.00 & 0.04 & -3.15 & 0.13 & -0.94 & 0.07 \\
\hline Ti I & 16 & & 2.54 & 0.05 & -2.41 & 0.24 & -0.19 & 0.08 \\
\hline Ti II & 36 & & 2.67 & 0.02 & -2.29 & 0.13 & -0.08 & 0.07 \\
\hline $\mathrm{Cr} \mathrm{I}$ & 15 & & 3.31 & 0.04 & -2.33 & 0.22 & -0.11 & 0.06 \\
\hline Cr II & 2 & & 3.66 & 0.07 & -1.98 & 0.14 & 0.22 & 0.09 \\
\hline Mn I & 6 & & 2.53 & 0.06 & -2.90 & 0.16 & -0.68 & 0.07 \\
\hline Fe I & 169 & & 5.28 & 0.01 & -2.22 & 0.18 & 0.00 & 0.02 \\
\hline Fe II & 21 & & 5.30 & 0.04 & -2.20 & 0.13 & 0.00 & 0.05 \\
\hline Co I & 5 & & 2.60 & 0.11 & -2.39 & 0.22 & -0.16 & 0.11 \\
\hline Ni I & 8 & & 3.75 & 0.04 & -2.47 & 0.16 & -0.25 & 0.06 \\
\hline $\mathrm{Zn} \mathrm{I}$ & 2 & & 2.21 & 0.14 & -2.35 & 0.16 & -0.13 & 0.20 \\
\hline Sr II & 2 & & -0.71 & 0.23 & -3.58 & 0.39 & -1.38 & 0.32 \\
\hline Ba II & 2 & & -2.58 & 0.13 & -4.76 & 0.21 & -2.55 & 0.20 \\
\hline $\mathrm{C}-\mathrm{H}$ & 2 & & 6.16 & 0.16 & -2.27 & 0.35 & -0.05 & 0.22 \\
\hline $\mathrm{C}-\mathrm{N}$ & 1 & & 6.04 & 0.65 & -1.79 & 0.78 & 0.43 & 0.70 \\
\hline O I & 1 & $<$ & 7.59 & $\cdots$ & -1.09 & $\cdots$ & 1.13 & $\cdots$ \\
\hline K I & 1 & $<$ & 2.75 & $\cdots$ & -2.28 & $\cdots$ & -0.06 & $\cdots$ \\
\hline $\mathrm{Cu} \mathrm{I}$ & 1 & $<$ & 1.83 & $\cdots$ & -2.36 & $\cdots$ & -0.14 & $\cdots$ \\
\hline Eu II & 1 & $<$ & -1.89 & $\cdots$ & -2.41 & $\cdots$ & -0.21 & $\cdots$ \\
\hline \multicolumn{9}{|c|}{ CarII-2064 } \\
\hline $\mathrm{Na} \mathrm{I}$ & 2 & & 4.21 & 0.15 & -2.03 & 0.33 & 0.33 & 0.20 \\
\hline $\mathrm{Mg} \mathrm{I}$ & 5 & & 5.62 & 0.12 & -1.98 & 0.22 & 0.38 & 0.13 \\
\hline $\mathrm{Al} \mathrm{I}$ & 2 & & 3.37 & 0.40 & -3.08 & 0.44 & -0.73 & 0.41 \\
\hline Si I & 2 & & 6.27 & 0.48 & -1.24 & 0.58 & 1.11 & 0.51 \\
\hline K I & 1 & & 3.46 & 0.18 & -1.57 & 0.23 & 0.79 & 0.18 \\
\hline $\mathrm{Ca} \mathrm{I}$ & 14 & & 4.48 & 0.06 & -1.86 & 0.15 & 0.49 & 0.09 \\
\hline Sc II & 6 & & 0.96 & 0.12 & -2.19 & 0.19 & 0.12 & 0.13 \\
\hline Ti I & 11 & & 3.43 & 0.05 & -1.52 & 0.23 & 0.83 & 0.06 \\
\hline Ti II & 26 & & 3.23 & 0.05 & -1.72 & 0.17 & 0.59 & 0.10 \\
\hline Cr I & 3 & & 2.73 & 0.35 & -2.91 & 0.44 & -0.55 & 0.36 \\
\hline Cr II & 1 & & 3.78 & 0.18 & -1.86 & 0.23 & 0.44 & 0.20 \\
\hline Mn I & 3 & & 3.02 & 0.16 & -2.42 & 0.26 & -0.06 & 0.17 \\
\hline Fe I & 81 & & 5.15 & 0.03 & -2.35 & 0.20 & 0.00 & 0.04 \\
\hline Fe II & 10 & & 5.19 & 0.06 & -2.31 & 0.15 & 0.00 & 0.09 \\
\hline Co I & 1 & & 2.43 & 0.28 & -2.56 & 0.35 & -0.20 & 0.29 \\
\hline $\mathrm{Ni} \mathrm{I}$ & 2 & & 4.31 & 0.17 & -1.91 & 0.24 & 0.44 & 0.17 \\
\hline Sr II & 2 & & -1.08 & 0.20 & -3.95 & 0.26 & -1.64 & 0.22 \\
\hline Ba II & 2 & & -0.97 & 0.11 & -3.15 & 0.20 & -0.84 & 0.16 \\
\hline
\end{tabular}

Table 6 continued 
Table 6 (continued)

\begin{tabular}{|c|c|c|c|c|c|c|c|c|}
\hline El. & $N$ & & $\log \epsilon$ & $\sigma_{\text {stat }}$ & {$[\mathrm{X} / \mathrm{H}]$} & $\sigma_{[\mathrm{X} / \mathrm{H}]}$ & {$[\mathrm{X} / \mathrm{Fe}]$} & $\sigma_{[\mathrm{X} / \mathrm{Fe}]}$ \\
\hline $\mathrm{C}-\mathrm{H}$ & 2 & & 6.45 & 0.21 & -1.98 & 0.45 & 0.38 & 0.31 \\
\hline O I & 2 & $<$ & 8.73 & $\cdots$ & 0.04 & $\cdots$ & 2.40 & $\cdots$ \\
\hline $\mathrm{Cu} \mathrm{I}$ & 2 & $<$ & 4.10 & $\cdots$ & -0.09 & $\ldots$ & 2.27 & $\cdots$ \\
\hline Zn I & 2 & $<$ & 2.76 & $\cdots$ & -1.80 & $\cdots$ & 0.55 & $\cdots$ \\
\hline Eu II & 2 & $<$ & -0.64 & $\cdots$ & -1.16 & $\cdots$ & 1.15 & $\cdots$ \\
\hline $\mathrm{C}-\mathrm{N}$ & 2 & $<$ & 4.48 & $\ldots$ & -3.35 & $\cdots$ & -1.00 & $\cdots$ \\
\hline \multicolumn{9}{|c|}{ CarII-4704 } \\
\hline $\mathrm{Na} \mathrm{I}$ & 2 & & 3.50 & 0.16 & -2.74 & 0.33 & -0.54 & 0.19 \\
\hline $\mathrm{Mg} \mathrm{I}$ & 5 & & 5.06 & 0.12 & -2.54 & 0.19 & -0.34 & 0.14 \\
\hline $\mathrm{Al} \mathrm{I}$ & 2 & & 3.00 & 0.56 & -3.45 & 0.58 & -1.25 & 0.58 \\
\hline Si I & 2 & & 4.96 & 0.41 & -2.55 & 0.47 & -0.35 & 0.41 \\
\hline K I & 1 & & 2.96 & 0.17 & -2.07 & 0.23 & 0.13 & 0.18 \\
\hline $\mathrm{Ca} \mathrm{I}$ & 14 & & 4.20 & 0.04 & -2.14 & 0.15 & 0.06 & 0.07 \\
\hline Sc II & 5 & & 0.74 & 0.08 & -2.41 & 0.15 & -0.22 & 0.10 \\
\hline Ti I & 5 & & 2.65 & 0.09 & -2.30 & 0.24 & -0.10 & 0.10 \\
\hline Ti II & 26 & & 2.65 & 0.05 & -2.30 & 0.14 & -0.12 & 0.09 \\
\hline Cr I & 9 & & 3.23 & 0.09 & -2.41 & 0.23 & -0.20 & 0.09 \\
\hline Mn I & 7 & & 2.58 & 0.09 & -2.85 & 0.18 & -0.64 & 0.10 \\
\hline $\mathrm{Fe} I$ & 105 & & 5.30 & 0.02 & -2.20 & 0.20 & 0.00 & 0.03 \\
\hline Fe II & 14 & & 5.32 & 0.04 & -2.19 & 0.12 & 0.00 & 0.05 \\
\hline Co I & 4 & & 2.69 & 0.15 & -2.30 & 0.24 & -0.10 & 0.15 \\
\hline Ni I & 3 & & 4.11 & 0.09 & -2.11 & 0.18 & 0.09 & 0.10 \\
\hline Sr II & 2 & & -1.68 & 0.26 & -4.55 & 0.31 & -2.36 & 0.27 \\
\hline Ba II & 2 & & -2.11 & 0.11 & -4.29 & 0.18 & -2.10 & 0.17 \\
\hline $\mathrm{C}-\mathrm{H}$ & 2 & & 5.63 & 0.16 & -2.80 & 0.35 & -0.60 & 0.22 \\
\hline O I & 2 & $<$ & 7.66 & $\cdots$ & -1.03 & $\cdots$ & 1.17 & $\cdots$ \\
\hline $\mathrm{Cu} \mathrm{I}$ & 2 & $<$ & 2.69 & $\cdots$ & -1.50 & $\cdots$ & 0.70 & $\cdots$ \\
\hline Zn I & 2 & $<$ & 2.88 & $\ldots$ & -1.69 & $\ldots$ & 0.52 & $\cdots$ \\
\hline Eu II & 2 & $<$ & -1.44 & $\ldots$ & -1.96 & $\cdots$ & 0.23 & $\ldots$ \\
\hline $\mathrm{C}-\mathrm{N}$ & 2 & $<$ & 5.75 & $\cdots$ & -2.08 & $\cdots$ & 0.13 & $\cdots$ \\
\hline \multicolumn{9}{|c|}{ CarII-4928 } \\
\hline $\mathrm{Na} \mathrm{I}$ & 2 & & 3.45 & 0.16 & -2.79 & 0.35 & 0.26 & 0.20 \\
\hline Mg I & 5 & & 5.25 & 0.12 & -2.35 & 0.21 & 0.71 & 0.16 \\
\hline $\mathrm{Al} \mathrm{I}$ & 2 & & 3.46 & 0.35 & -2.99 & 0.45 & 0.07 & 0.40 \\
\hline Si I & 2 & & 4.81 & 0.31 & -2.70 & 0.40 & 0.35 & 0.31 \\
\hline $\mathrm{Ca} \mathrm{I}$ & 2 & & 4.08 & 0.18 & -2.26 & 0.28 & 0.80 & 0.20 \\
\hline Sc II & 6 & & 0.36 & 0.11 & -2.79 & 0.23 & 0.24 & 0.19 \\
\hline Ti II & 22 & & 2.58 & 0.09 & -2.37 & 0.22 & 0.66 & 0.18 \\
\hline Cr I & 3 & & 1.90 & 0.20 & -3.74 & 0.37 & -0.68 & 0.21 \\
\hline Mn I & 3 & & 1.47 & 0.16 & -3.96 & 0.30 & -0.91 & 0.17 \\
\hline Fe I & 46 & & 4.44 & 0.04 & -3.06 & 0.26 & 0.00 & 0.05 \\
\hline Fe II & 5 & & 4.47 & 0.14 & -3.03 & 0.23 & 0.00 & 0.20 \\
\hline Co I & 2 & & 2.09 & 0.24 & -2.90 & 0.39 & 0.16 & 0.26 \\
\hline Sr II & 2 & & -1.99 & 0.25 & -4.86 & 0.33 & -1.83 & 0.30 \\
\hline $\mathrm{C}-\mathrm{H}$ & 2 & & 5.67 & 0.26 & -2.77 & 0.56 & 0.29 & 0.39 \\
\hline O I & 2 & $<$ & 8.50 & $\ldots$ & -0.19 & $\ldots$ & 2.86 & $\ldots$ \\
\hline
\end{tabular}

Table 6 continued 
Table 6 (continued)

\begin{tabular}{|c|c|c|c|c|c|c|c|c|}
\hline El. & $N$ & & $\log \epsilon$ & $\sigma_{\text {stat }}$ & {$[\mathrm{X} / \mathrm{H}]$} & $\sigma_{[\mathrm{X} / \mathrm{H}]}$ & {$[\mathrm{X} / \mathrm{Fe}]$} & $\sigma_{[\mathrm{X} / \mathrm{Fe}]}$ \\
\hline K I & 2 & $<$ & 3.04 & $\cdots$ & -1.99 & $\ldots$ & 1.06 & $\ldots$ \\
\hline $\mathrm{Ni} \mathrm{I}$ & 2 & $<$ & 5.16 & $\ldots$ & -1.06 & $\cdots$ & 1.99 & $\cdots$ \\
\hline $\mathrm{Cu} \mathrm{I}$ & 2 & $<$ & 3.06 & $\cdots$ & -1.13 & $\cdots$ & 1.93 & $\cdots$ \\
\hline Zn I & 2 & $<$ & 3.08 & $\ldots$ & -1.48 & $\ldots$ & 1.58 & $\ldots$ \\
\hline Ba II & 2 & $<$ & -1.60 & $\ldots$ & -3.78 & $\cdots$ & -0.74 & $\cdots$ \\
\hline $\mathrm{Eu} \mathrm{II}$ & 2 & $<$ & -1.04 & $\cdots$ & -1.56 & $\ldots$ & 1.47 & $\ldots$ \\
\hline $\mathrm{C}-\mathrm{N}$ & 2 & $<$ & 6.97 & $\cdots$ & -0.86 & $\ldots$ & 2.19 & $\ldots$ \\
\hline \multicolumn{9}{|c|}{ CarII-5664 } \\
\hline $\mathrm{Na} \mathrm{I}$ & 2 & & 3.37 & 0.12 & -2.87 & 0.27 & 0.66 & 0.17 \\
\hline $\mathrm{Mg} \mathrm{I}$ & 7 & & 4.80 & 0.04 & -2.79 & 0.12 & 0.73 & 0.09 \\
\hline Al I & 2 & & 2.46 & 0.17 & -3.99 & 0.29 & -0.46 & 0.27 \\
\hline Si I & 2 & & 4.59 & 0.13 & -2.92 & 0.26 & 0.61 & 0.14 \\
\hline K I & 2 & & 2.52 & 0.07 & -2.51 & 0.16 & 1.02 & 0.09 \\
\hline $\mathrm{Ca} \mathrm{I}$ & 12 & & 3.18 & 0.03 & -3.16 & 0.13 & 0.36 & 0.07 \\
\hline Sc II & 8 & & -0.14 & 0.06 & -3.29 & 0.12 & 0.24 & 0.08 \\
\hline Ti I & 6 & & 1.64 & 0.04 & -3.31 & 0.22 & 0.21 & 0.07 \\
\hline Ti II & 32 & & 1.68 & 0.03 & -3.27 & 0.11 & 0.27 & 0.07 \\
\hline Cr I & 3 & & 1.47 & 0.09 & -4.17 & 0.25 & -0.64 & 0.12 \\
\hline Mn I & 3 & & 0.91 & 0.11 & -4.52 & 0.25 & -0.99 & 0.12 \\
\hline Fe I & 113 & & 3.97 & 0.01 & -3.53 & 0.18 & 0.00 & 0.02 \\
\hline Fe II & 10 & & 3.96 & 0.04 & -3.54 & 0.10 & 0.00 & 0.05 \\
\hline Co I & 4 & & 1.47 & 0.07 & -3.52 & 0.22 & 0.00 & 0.08 \\
\hline $\mathrm{Ni} \mathrm{I}$ & 1 & & 2.25 & 0.20 & -3.96 & 0.28 & -0.44 & 0.21 \\
\hline Sr II & 2 & & -2.86 & 0.09 & -5.73 & 0.16 & -2.19 & 0.12 \\
\hline $\mathrm{C}-\mathrm{H}$ & 2 & & 4.74 & 0.21 & -3.69 & 0.48 & -0.17 & 0.33 \\
\hline O I & 2 & $<$ & 6.88 & $\ldots$ & -1.81 & $\ldots$ & 1.72 & $\ldots$ \\
\hline $\mathrm{Cu} \mathrm{I}$ & 2 & $<$ & 1.47 & $\ldots$ & -2.72 & $\cdots$ & 0.80 & $\cdots$ \\
\hline Zn I & 2 & $<$ & 1.54 & $\ldots$ & -3.02 & $\ldots$ & 0.50 & $\ldots$ \\
\hline $\mathrm{Ba}$ II & 2 & $<$ & -3.29 & $\ldots$ & -5.47 & $\ldots$ & -1.93 & $\ldots$ \\
\hline Eu II & 2 & $<$ & -2.81 & $\ldots$ & -3.33 & $\cdots$ & 0.21 & $\cdots$ \\
\hline $\mathrm{C}-\mathrm{N}$ & 2 & $<$ & 5.79 & $\ldots$ & -2.04 & $\ldots$ & 1.48 & $\ldots$ \\
\hline \multicolumn{9}{|c|}{ CarII-6544 } \\
\hline O I & 2 & & 6.99 & 0.09 & -1.70 & 0.18 & 0.96 & 0.21 \\
\hline $\mathrm{Na} \mathrm{I}$ & 2 & & 3.53 & 0.20 & -2.71 & 0.44 & -0.05 & 0.28 \\
\hline Mg I & 7 & & 5.11 & 0.08 & -2.49 & 0.16 & 0.17 & 0.10 \\
\hline $\mathrm{Al} \mathrm{I}$ & 2 & & 3.17 & 0.25 & -3.28 & 0.36 & -0.62 & 0.26 \\
\hline Si I & 2 & & 5.16 & 0.25 & -2.35 & 0.36 & 0.31 & 0.26 \\
\hline K I & 1 & & 2.69 & 0.20 & -2.34 & 0.28 & 0.32 & 0.20 \\
\hline $\mathrm{Ca} \mathrm{I}$ & 21 & & 3.78 & 0.03 & -2.56 & 0.15 & 0.10 & 0.07 \\
\hline Sc II & 11 & & 0.33 & 0.04 & -2.82 & 0.11 & -0.16 & 0.06 \\
\hline Ti I & 22 & & 2.19 & 0.04 & -2.77 & 0.29 & -0.11 & 0.10 \\
\hline Ti II & 44 & & 2.34 & 0.02 & -2.61 & 0.12 & 0.05 & 0.08 \\
\hline Cr I & 16 & & 2.84 & 0.03 & -2.80 & 0.27 & -0.15 & 0.07 \\
\hline Cr II & 1 & & 3.12 & 0.14 & -2.52 & 0.19 & 0.14 & 0.15 \\
\hline Mn I & 7 & & 2.29 & 0.06 & -3.14 & 0.22 & -0.48 & 0.07 \\
\hline $\mathrm{Fe} \mathrm{I}$ & 144 & & 4.84 & 0.01 & -2.66 & 0.21 & 0.00 & 0.02 \\
\hline
\end{tabular}

Table 6 continued 
Table 6 (continued)

\begin{tabular}{|c|c|c|c|c|c|c|c|c|}
\hline El. & $N$ & & $\log \epsilon$ & $\sigma_{\text {stat }}$ & {$[\mathrm{X} / \mathrm{H}]$} & $\sigma_{[\mathrm{X} / \mathrm{H}]}$ & {$[\mathrm{X} / \mathrm{Fe}]$} & $\sigma_{[\mathrm{X} / \mathrm{Fe}]}$ \\
\hline Fe II & 22 & & 4.84 & 0.03 & -2.66 & 0.13 & 0.00 & 0.05 \\
\hline Co I & 5 & & 2.19 & 0.12 & -2.80 & 0.27 & -0.14 & 0.13 \\
\hline $\mathrm{Ni} \mathrm{I}$ & 12 & & 3.43 & 0.04 & -2.79 & 0.20 & -0.13 & 0.05 \\
\hline Zn I & 1 & & 1.47 & 0.13 & -3.09 & 0.15 & -0.43 & 0.23 \\
\hline Sr II & 2 & & -1.58 & 0.15 & -4.45 & 0.25 & -1.79 & 0.21 \\
\hline Ba II & 4 & & -2.00 & 0.10 & -4.18 & 0.16 & -1.52 & 0.15 \\
\hline $\mathrm{C}-\mathrm{H}$ & 2 & & 5.24 & 0.16 & -3.19 & 0.35 & -0.54 & 0.21 \\
\hline $\mathrm{C}-\mathrm{N}$ & 1 & & 5.48 & 0.54 & -2.35 & 0.67 & 0.31 & 0.58 \\
\hline $\mathrm{Cu} \mathrm{I}$ & 1 & $<$ & 1.48 & $\cdots$ & -2.71 & $\cdots$ & -0.06 & $\cdots$ \\
\hline Eu II & 1 & $<$ & -2.34 & $\cdots$ & -2.86 & $\cdots$ & -0.20 & $\cdots$ \\
\hline \multicolumn{9}{|c|}{ CarII-7872 } \\
\hline O I & 2 & & 7.56 & 0.07 & -1.13 & 0.15 & 1.38 & 0.21 \\
\hline $\mathrm{Na} \mathrm{I}$ & 2 & & 3.21 & 0.18 & -3.03 & 0.39 & -0.52 & 0.23 \\
\hline $\mathrm{Mg} \mathrm{I}$ & 5 & & 5.09 & 0.06 & -2.51 & 0.20 & -0.00 & 0.08 \\
\hline $\mathrm{Al} \mathrm{I}$ & 2 & & 3.59 & 0.27 & -2.86 & 0.43 & -0.35 & 0.31 \\
\hline Si I & 2 & & 5.17 & 0.32 & -2.34 & 0.43 & 0.17 & 0.34 \\
\hline K I & 2 & & 2.83 & 0.12 & -2.20 & 0.25 & 0.31 & 0.12 \\
\hline $\mathrm{Ca} \mathrm{I}$ & 12 & & 3.83 & 0.04 & -2.51 & 0.17 & 0.00 & 0.07 \\
\hline Sc II & 5 & & 0.54 & 0.06 & -2.61 & 0.16 & -0.12 & 0.08 \\
\hline Ti I & 18 & & 2.38 & 0.04 & -2.57 & 0.30 & -0.06 & 0.10 \\
\hline Ti II & 27 & & 2.55 & 0.03 & -2.40 & 0.12 & 0.09 & 0.09 \\
\hline Cr I & 16 & & 2.86 & 0.04 & -2.78 & 0.27 & -0.27 & 0.07 \\
\hline Mn I & 6 & & 2.29 & 0.08 & -3.13 & 0.20 & -0.62 & 0.10 \\
\hline $\mathrm{Fe} I$ & 123 & & 4.99 & 0.02 & -2.51 & 0.21 & 0.00 & 0.02 \\
\hline Fe II & 20 & & 5.01 & 0.04 & -2.49 & 0.13 & 0.00 & 0.06 \\
\hline Co I & 5 & & 2.36 & 0.10 & -2.63 & 0.22 & -0.12 & 0.11 \\
\hline Ni I & 10 & & 3.62 & 0.05 & -2.60 & 0.20 & -0.08 & 0.06 \\
\hline Zn I & 1 & & 1.73 & 0.10 & -2.83 & 0.12 & -0.32 & 0.23 \\
\hline Sr II & 1 & & -1.03 & 0.36 & -3.90 & 0.46 & -1.41 & 0.41 \\
\hline Ba II & 5 & & -0.89 & 0.06 & -3.07 & 0.16 & -0.57 & 0.13 \\
\hline $\mathrm{C}-\mathrm{H}$ & 2 & & 6.24 & 0.14 & -2.19 & 0.31 & 0.32 & 0.20 \\
\hline $\mathrm{C}-\mathrm{N}$ & 1 & & 6.48 & 0.53 & -1.35 & 0.55 & 1.17 & 0.55 \\
\hline $\mathrm{Cu} \mathrm{I}$ & 1 & $<$ & 1.42 & $\cdots$ & -2.77 & $\cdots$ & -0.25 & $\cdots$ \\
\hline Eu II & 1 & $<$ & -2.59 & $\cdots$ & -3.11 & $\cdots$ & -0.61 & $\cdots$ \\
\hline \multicolumn{9}{|c|}{ CarII-9296 } \\
\hline $\mathrm{Na} \mathrm{I}$ & 2 & & 3.38 & 0.15 & -2.86 & 0.33 & 0.03 & 0.19 \\
\hline Mg I & 5 & & 5.18 & 0.09 & -2.42 & 0.21 & 0.47 & 0.11 \\
\hline $\mathrm{Al} \mathrm{I}$ & 2 & & 3.42 & 0.35 & -3.04 & 0.44 & -0.15 & 0.40 \\
\hline Si I & 2 & & 4.71 & 0.28 & -2.80 & 0.39 & 0.09 & 0.29 \\
\hline $\mathrm{Ca}$ I & 12 & & 3.82 & 0.05 & -2.52 & 0.17 & 0.37 & 0.09 \\
\hline Sc II & 6 & & 0.37 & 0.07 & -2.78 & 0.18 & 0.13 & 0.11 \\
\hline Ti I & 4 & & 2.60 & 0.15 & -2.35 & 0.30 & 0.54 & 0.16 \\
\hline Ti II & 18 & & 2.49 & 0.05 & -2.46 & 0.18 & 0.45 & 0.12 \\
\hline Cr I & 4 & & 2.28 & 0.15 & -3.36 & 0.35 & -0.47 & 0.18 \\
\hline Mn I & 3 & & 2.22 & 0.36 & -3.21 & 0.42 & -0.32 & 0.36 \\
\hline $\mathrm{Fe} I$ & 72 & & 4.61 & 0.03 & -2.89 & 0.23 & 0.00 & 0.05 \\
\hline
\end{tabular}

Table 6 continued 
Table 6 (continued)

\begin{tabular}{|c|c|c|c|c|c|c|c|c|}
\hline El. & $N$ & & $\log \epsilon$ & $\sigma_{\text {stat }}$ & {$[\mathrm{X} / \mathrm{H}]$} & $\sigma_{[\mathrm{X} / \mathrm{H}]}$ & {$[\mathrm{X} / \mathrm{Fe}]$} & $\sigma_{[\mathrm{X} / \mathrm{Fe}]}$ \\
\hline Fe II & 8 & & 4.59 & 0.08 & -2.91 & 0.17 & 0.00 & 0.11 \\
\hline Co I & 2 & & 1.76 & 0.21 & -3.23 & 0.36 & -0.34 & 0.23 \\
\hline $\mathrm{Ni} \mathrm{I}$ & 1 & & 3.24 & 0.34 & -2.98 & 0.42 & -0.09 & 0.34 \\
\hline Sr II & 2 & & -1.28 & 0.26 & -4.15 & 0.39 & -1.25 & 0.32 \\
\hline Ba II & 3 & & -1.95 & 0.14 & -4.13 & 0.25 & -1.23 & 0.22 \\
\hline $\mathrm{C}-\mathrm{H}$ & 2 & & 5.72 & 0.32 & -2.71 & 0.56 & 0.17 & 0.41 \\
\hline O I & 2 & $<$ & 7.88 & $\ldots$ & -0.81 & $\ldots$ & 2.08 & $\cdots$ \\
\hline K I & 2 & $<$ & 3.13 & $\cdots$ & -1.90 & $\cdots$ & 0.99 & $\cdots$ \\
\hline $\mathrm{Cu} \mathrm{I}$ & 2 & $<$ & 2.38 & $\ldots$ & -1.81 & $\ldots$ & 1.07 & $\cdots$ \\
\hline $\mathrm{Zn} \mathrm{I}$ & 2 & $<$ & 2.70 & $\cdots$ & -1.86 & $\cdots$ & 1.03 & $\cdots$ \\
\hline Eu II & 2 & $<$ & -1.44 & $\cdots$ & -1.96 & $\cdots$ & 0.95 & $\cdots$ \\
\hline $\mathrm{C}-\mathrm{N}$ & 2 & $<$ & 6.31 & $\ldots$ & -1.52 & $\cdots$ & 1.37 & $\cdots$ \\
\hline \multicolumn{9}{|c|}{ CarII-V3 } \\
\hline $\mathrm{Na} \mathrm{I}$ & 2 & & 3.04 & 0.13 & -3.20 & 0.27 & -0.56 & 0.15 \\
\hline $\mathrm{Mg} \mathrm{I}$ & 2 & & 5.62 & 0.31 & -1.98 & 0.40 & 0.67 & 0.33 \\
\hline Al I & 2 & & 3.24 & 0.16 & -3.21 & 0.32 & -0.56 & 0.17 \\
\hline Si I & 1 & & 4.29 & 0.31 & -3.22 & 0.41 & -0.57 & 0.31 \\
\hline $\mathrm{Ca} \mathrm{I}$ & 1 & & 4.01 & 0.36 & -2.33 & 0.49 & 0.31 & 0.38 \\
\hline Sc II & 2 & & 0.66 & 0.17 & -2.49 & 0.26 & 0.23 & 0.21 \\
\hline Ti II & 14 & & 2.53 & 0.05 & -2.42 & 0.18 & 0.30 & 0.11 \\
\hline Cr I & 3 & & 2.88 & 0.13 & -2.76 & 0.35 & -0.12 & 0.14 \\
\hline $\mathrm{Fe} I$ & 22 & & 4.85 & 0.04 & -2.65 & 0.28 & 0.00 & 0.06 \\
\hline Fe II & 8 & & 4.78 & 0.06 & -2.72 & 0.13 & 0.00 & 0.09 \\
\hline \multicolumn{9}{|c|}{ CarIII-1120 } \\
\hline $\mathrm{Na} \mathrm{I}$ & 2 & & 3.75 & 0.20 & -2.49 & 0.45 & 1.39 & 0.26 \\
\hline $\mathrm{Mg} \mathrm{I}$ & 5 & & 4.94 & 0.10 & -2.66 & 0.23 & 1.22 & 0.13 \\
\hline $\mathrm{Al} \mathrm{I}$ & 2 & & 2.41 & 0.37 & -4.04 & 0.46 & -0.17 & 0.45 \\
\hline Si I & 1 & & 4.72 & 0.42 & -2.79 & 0.50 & 1.08 & 0.42 \\
\hline $\mathrm{Ca} \mathrm{I}$ & 1 & & 2.93 & 0.22 & -3.41 & 0.28 & 0.46 & 0.24 \\
\hline Sc II & 5 & & -0.60 & 0.12 & -3.75 & 0.18 & 0.14 & 0.16 \\
\hline Ti II & 6 & & 1.36 & 0.07 & -3.59 & 0.18 & 0.30 & 0.14 \\
\hline Cr I & 2 & & 1.29 & 0.17 & -4.34 & 0.33 & -0.47 & 0.18 \\
\hline Mn I & 3 & & 0.86 & 0.18 & -4.57 & 0.25 & -0.70 & 0.22 \\
\hline $\mathrm{Fe} \mathrm{I}$ & 48 & & 3.63 & 0.03 & -3.87 & 0.27 & 0.00 & 0.05 \\
\hline Fe II & 4 & & 3.61 & 0.08 & -3.89 & 0.14 & 0.00 & 0.12 \\
\hline Co I & 3 & & 1.62 & 0.17 & -3.37 & 0.28 & 0.51 & 0.20 \\
\hline Sr II & 2 & & -2.75 & 0.24 & -5.62 & 0.28 & -1.72 & 0.26 \\
\hline C-H & 2 & & 5.81 & 0.28 & -2.62 & 0.63 & 1.25 & 0.43 \\
\hline O I & 2 & $<$ & 7.50 & $\ldots$ & -1.19 & $\ldots$ & 2.68 & $\ldots$ \\
\hline K I & 2 & $<$ & 2.31 & $\ldots$ & -2.72 & $\ldots$ & 1.15 & $\ldots$ \\
\hline $\mathrm{Ni} \mathrm{I}$ & 2 & $<$ & 3.12 & $\ldots$ & -3.10 & $\ldots$ & 0.78 & $\ldots$ \\
\hline $\mathrm{Cu} \mathrm{I}$ & 2 & $<$ & 2.07 & $\ldots$ & -2.12 & $\ldots$ & 1.75 & $\ldots$ \\
\hline $\mathrm{Zn} \mathrm{I}$ & 2 & $<$ & 2.55 & $\ldots$ & -2.01 & $\ldots$ & 1.86 & $\ldots$ \\
\hline Ba II & 2 & $<$ & -2.48 & $\ldots$ & -4.66 & $\ldots$ & -0.76 & $\ldots$ \\
\hline Eu II & 2 & $<$ & -1.82 & $\ldots$ & -2.34 & $\ldots$ & 1.55 & $\ldots$ \\
\hline $\mathrm{C}-\mathrm{N}$ & 2 & $<$ & 5.15 & $\ldots$ & -2.68 & $\ldots$ & 1.20 & $\ldots$ \\
\hline
\end{tabular}

Table 6 continued 
Table 6 (continued)

\begin{tabular}{|c|c|c|c|c|c|c|c|c|}
\hline El. & $N$ & & $\log \epsilon$ & $\sigma_{\text {stat }}$ & {$[\mathrm{X} / \mathrm{H}]$} & $\sigma_{[\mathrm{X} / \mathrm{H}]}$ & {$[\mathrm{X} / \mathrm{Fe}]$} & $\sigma_{[\mathrm{X} / \mathrm{Fe}]}$ \\
\hline \multicolumn{9}{|c|}{ CarIII-8144 } \\
\hline $\mathrm{Na} \mathrm{I}$ & 2 & & 3.97 & 0.14 & -2.27 & 0.31 & -0.00 & 0.19 \\
\hline $\mathrm{Mg} \mathrm{I}$ & 7 & & 5.58 & 0.08 & -2.02 & 0.18 & 0.25 & 0.10 \\
\hline $\mathrm{Al} \mathrm{I}$ & 2 & & 3.58 & 0.23 & -2.87 & 0.27 & -0.60 & 0.24 \\
\hline Si I & 2 & & 5.84 & 0.18 & -1.67 & 0.32 & 0.60 & 0.21 \\
\hline K I & 1 & & 3.86 & 0.21 & -1.17 & 0.29 & 1.09 & 0.22 \\
\hline $\mathrm{Ca} \mathrm{I}$ & 24 & & 4.62 & 0.03 & -1.72 & 0.14 & 0.55 & 0.05 \\
\hline Sc II & 5 & & 1.42 & 0.07 & -1.73 & 0.17 & 0.50 & 0.10 \\
\hline Ti I & 15 & & 3.05 & 0.02 & -1.90 & 0.21 & 0.36 & 0.05 \\
\hline Ti II & 38 & & 3.14 & 0.03 & -1.81 & 0.14 & 0.43 & 0.08 \\
\hline Cr I & 13 & & 3.23 & 0.05 & -2.41 & 0.20 & -0.14 & 0.06 \\
\hline Cr II & 2 & & 3.67 & 0.08 & -1.97 & 0.14 & 0.26 & 0.10 \\
\hline Mn I & 7 & & 2.97 & 0.11 & -2.46 & 0.19 & -0.19 & 0.12 \\
\hline $\mathrm{Fe} I$ & 147 & & 5.23 & 0.01 & -2.27 & 0.18 & 0.00 & 0.02 \\
\hline Fe II & 16 & & 5.27 & 0.04 & -2.23 & 0.13 & 0.00 & 0.06 \\
\hline Co I & 3 & & 2.56 & 0.14 & -2.43 & 0.24 & -0.16 & 0.16 \\
\hline Ni I & 4 & & 3.85 & 0.08 & -2.37 & 0.19 & -0.10 & 0.09 \\
\hline Zn I & 2 & & 2.84 & 0.08 & -1.72 & 0.12 & 0.55 & 0.15 \\
\hline Sr II & 2 & & -0.75 & 0.26 & -3.62 & 0.38 & -1.39 & 0.32 \\
\hline Ba II & 1 & & -2.07 & 0.14 & -4.25 & 0.18 & -2.02 & 0.17 \\
\hline $\mathrm{C}-\mathrm{H}$ & 2 & & 6.31 & 0.16 & -2.12 & 0.35 & 0.15 & 0.23 \\
\hline O I & 2 & $<$ & 8.17 & $\ldots$ & -0.52 & $\ldots$ & 1.74 & $\ldots$ \\
\hline $\mathrm{Cu} \mathrm{I}$ & 2 & $<$ & 2.50 & $\ldots$ & -1.69 & $\ldots$ & 0.58 & $\ldots$ \\
\hline Eu II & 2 & $<$ & -1.30 & $\ldots$ & -1.82 & $\cdots$ & 0.42 & $\cdots$ \\
\hline $\mathrm{C}-\mathrm{N}$ & 2 & $<$ & 6.03 & $\ldots$ & -1.80 & $\ldots$ & 0.46 & $\ldots$ \\
\hline
\end{tabular}

